\title{
Condiciones de cristalización y diferenciación de las lavas del volcán El Metate (Campo Volcánico de Michoacán- Guanajuato, México)
}

\section{Crystallization conditions during the differentiation of the El Metate volcano lavas (Michoacán-Guanajuato Volcanic Field, México)}

\author{
E. Losantos ${ }^{1}$, J.M. Cebriá1, D.J. Morán-Zenteno², B.M. Martiny², J. López-Ruiz \\ 1 Instituto de Geociencias (CSIC, UCM). José Gutiérrez Abascal, 2. 28006 Madrid. España. Email: e.losantos@csic.es \\ 2 Instituto de Geología (UNAM). Ciudad Universitaria. 04510 México, D.F. México
}

\section{RESUMEN}

El Metate es un volcán en escudo situado en el sector sur del Campo Volcánico de Michoacán-Guanajuato, uno de los dos campos volcánicos más grandes del Cinturón Volcánico Transmexicano. Su actividad tuvo lugar aproximadamente $4.700 \pm 200$ a B.P y produjo más de quince coladas de afinidad calcoalcalina que muestran diferente grado de diferenciación.

Las temperaturas calculadas mediante geotermómetros mineral-líquido para olivino, plagioclasa, y piroxenos muestran que la fase más temprana en cristalizar fue el olivino $\left(1232-1198{ }^{\circ} \mathrm{C}\right)$, seguido de plagioclasa $\left(1162-1126^{\circ} \mathrm{C}\right)$, ortopiroxeno $\left(1147-1027^{\circ} \mathrm{C}\right)$ y clinopiroxeno $\left(1147-1018^{\circ} \mathrm{C}\right)$. Las estimaciones de presión sugieren que la cristalización comenzó a $\sim 7$ kbar y continuó hasta niveles superficiales. El contenido en agua del fundido durante la cristalización de la plagioclasa fue de $\sim 1.6 \%$ en peso.

Las temperaturas calculadas a partir del contenido en Al de los anfíboles, indican que cristalizaron entre 995 y $922{ }^{\circ} \mathrm{C}$, a una presión media de $3.5 \mathrm{kbar}$ y con un contenido en $\mathrm{H}_{2} \mathrm{O}$ del fundido de entre $5.2 \%$ y $6.9 \%$. Aunque estos valores estarían de acuerdo con que el anfíbol representa una fase tardía en el proceso de cristalización, el que estos cristales presenten siempre texturas de desequilibrio, que se observan también de forma ocasional en otras fases, sugiere que pueden representar xenocristales y/o que la cristalización de estas lavas ha tenido lugar en sistema abierto.

Palabras clave: Geotermobarometría; cristalización fraccionada; química mineral; diferenciación magmática.

\section{ABSTRACT}

El Metate is a shield volcano located in the southern sector of the Michoacan-Guanajuato Volcanic Field, one of two largest monogenetic volcanic fields of the Transmexican Volcanic Belt. It was active c. $4.700 \pm 200$ years B.P and emitted about fifteen calcalkaline lava flows showing variable differentiation degrees.

Temperatures calculated from mineral-liquid geothermobarometers for olivine, plagioclase and pyroxene, suggest that olivine was the earliest fractionating phase $\left(1232-1198{ }^{\circ} \mathrm{C}\right)$, followed by plagioclase $\left(1162-1126{ }^{\circ} \mathrm{C}\right)$,

Recibido el 16 de junio de 2014 / Aceptado el 10 de noviembre de 2014 / Publicado online el 17 de diciembre de 2014

Citation / Cómo citar este artículo: E. Losantos et al. (2014). Condiciones de cristalización y diferenciación de las lavas del volcán El Metate (Campo Volcánico de Michoacán-Guanajuato, México). Estudios Geológicos 70(2): e020. http://dx.doi.org/10.3989/ egeol.41589.349.

Copyright: () 2014 CSIC. This is an open-access article distributed under the terms of the Creative Commons Attribution-Non Commercial (by-nc) Spain 3.0 License. 
orthopyroxene $\left(1147-1027^{\circ} \mathrm{C}\right)$ and clinopyroxene $\left(1147-1018^{\circ} \mathrm{C}\right)$. Pressure estimations indicate that crystallization started at $\sim 7 \mathrm{kbar}$ and progressed up to surface levels. Water contents in the melts during crystalliztion of plagioclase is estimated at $\sim 1.6 \%$.

Temperatures calculated on the basis of Al content in amphibole, provide a crystallization range between 995 and $922{ }^{\circ} \mathrm{C}$, at an average pressure of $3.5 \mathrm{kbar}$ and water contents between $5.2 \%$ and $6.9 \%$. Although these values could agree with a scenario where amphibole represents a late crystallization phase along the previous fractionating sequence, the systematic presence of disequilibrium textures, which are also observed occasionally in other phases, suggest that other possibilities such as open-system crystallization cannot be discarded.

Keywords: Geothermobarometry; fractional crystallization; mineral chemistry; magmatic differentiation.

\section{Introducción}

Para establecer la evolución de los fundidos desde su acumulación en la cámara magmática hasta su ascenso y extrusión, es necesario conocer la temperatura y la presión a la que se formaron las diferentes fases que han cristalizado en el magma. Una de las aproximaciones más frecuentes consiste en estimar dichos parámetros a partir de la composición de las fases minerales, mediante la aplicación de geotermobarómetros basados en equilibrios químicos mineral-mineral y mineral-líquido. En la actualidad la aplicación de esta metodología se ha facilitado mucho al contarse con un completo arsenal de calibraciones para las fases más comunes en sistemas ígneos y adaptadas a un amplio rango de condiciones de presión, temperatura y composición (ver Putirka, 2008).

En efecto, estudios recientes sobre geotermobarometría en magmas basálticos (ver p.ej. Costa et al., 2013; Dahren et al., 2012; Keiding \& Sigmarsson, 2012) han confirmado que este tipo de cálculos permite obtener información detallada sobre la evolución y funcionamiento del sistema magmático durante la diferenciación, incluidos aquellos que tienen lugar en sistema abierto, como la asimilación o la mezcla de magmas, y que son esenciales para perfilar cualquier modelo petrogenético. De hecho, como han demostrado otro tipo de estudios, contar con información sobre la evolución de la paragénesis mineral permite cuantificar con mayor precisión los procesos de diferenciación (ver p.ej. Cebriá et al., 2011).

Por estas razones, y en el contexto de una investigación más amplia sobre la petrogénesis del volcanismo en el Campo Volcánico de MichoacánGuanajuato (México) se ha realizado un estudio geotermobarométrico $\left(\mathrm{P}-\mathrm{T}-\mathrm{H}_{2} \mathrm{O}\right)$ a partir de la paragénesis mineral observada en un primer conjunto de
11 muestras representativas de las lavas emitidas por el volcán El Metate, como paso previo para establecer un modelo petrogenético cuantitativo que pueda ser aplicable a volcanes similares de la región. Las muestras fueron seleccionadas de modo que cubrieran la secuencia de lavas emitidas por el volcán. En este trabajo se detallan los resultados preliminares de dicha aproximación.

\section{Contexto geológico}

El Cinturón Volcánico Transmexicano (CVTM), es considerado el mayor arco volcánico Neógeno de Norteamérica, extendiéndose por la parte central del país en una franja entre las latitudes $18^{\circ} 30^{\prime} \mathrm{N}$ y $21^{\circ} 30^{\prime} \mathrm{N}$ sobre el extremo sur de la placa norteamericana (Fig. 1). Está constituido por campos monogenéticos, estratovolcanes y mesetas volcánicas cuya distribución está controlada por la tectónica regional (Ferrari et al., 1999; Ferrari et al., 2012; Johnson \& Harrison, 1989; Suter et al., 1999). La composición de las lavas muestra una gran variabilidad composicional, si bien predominan los términos calcoalcalinos (ver Ferrari et al., 2012; Gómez-Tuena et al., 2007 y referencias incluidas).

El Campo Volcánico de Michoacán-Guanajuato (CVMG) es uno de los dos campos monogenéticos más grandes del CVTM (Hasenaka, 1994; Hasenaka \& Carmichael, 1985). Se sitúa en la parte centro-occidental del mismo y comprende cerca de 400 volcanes de tamaño medio $(2-12 \mathrm{~km}$ de diámetro basal y 100-1000 m de altura; Hasenaka, 1994) y alrededor de 1000 afloramientos de menor entidad producidos por actividad monogenética, incluyendo maares, domos y conos de ceniza (Hasenaka \& Carmichael, 1987), entre los cuales pueden encontrarse $\sim 377$ pequeños volcanes en escudo andesíticos (Hasenaka, 1994) y más raramente estratovolcanes (e.g. Tancítaro, Patamban), distribuidos en una 

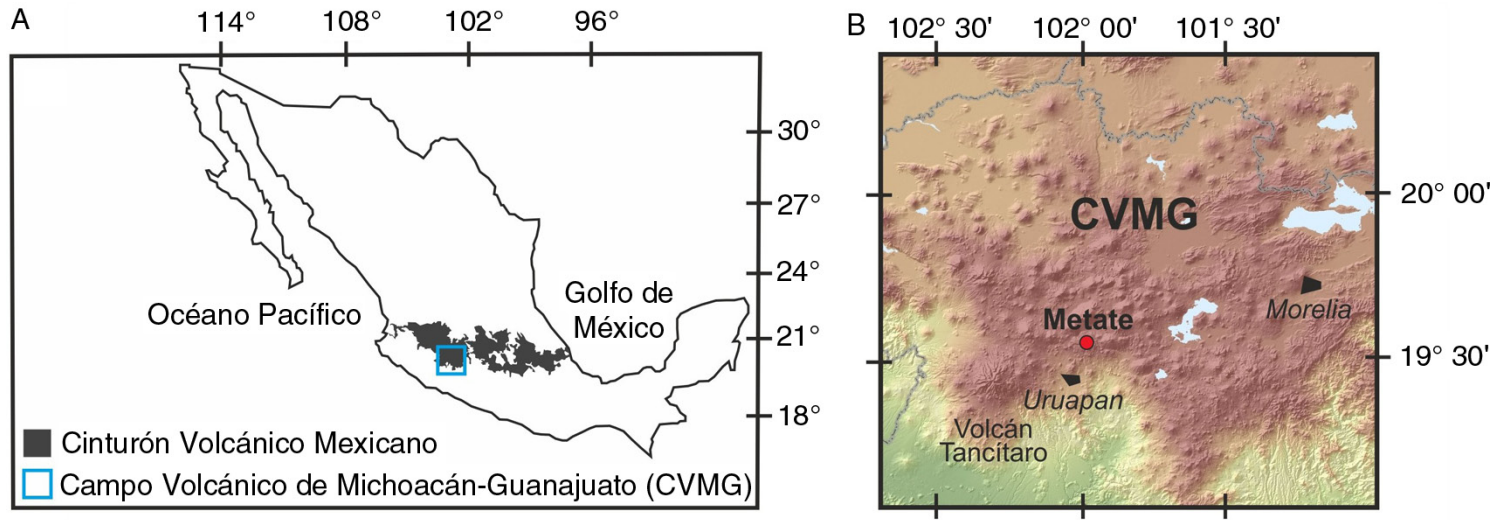

Fig. 1.-A) Situación del Cinturón Volcánico Mexicano (CVM) y del Campo Volcánico de Michoacán-Guanajuato (CVMG). B) Localización del volcán El Metate en el CVMG.

superficie de unos $40000 \mathrm{~km}^{2}$. Se considera que el volcanismo monogenético en esta zona empezó hace $\sim 3$ Ma (Hasenaka \& Carmichael, 1985), con una clara tendencia del frente volcánico a migrar hacia el suroeste. Predominan los términos calcoalcalinos, aunque también existen alcalinos y más raramente adakíticos (ver Ferrari et al., 2012; Gómez-Tuena et al., 2007 y referencias incluidas).

El Metate es un volcán en escudo que ocupa un área de algo más de $101 \mathrm{~km}^{2}$ en el sector sur del CVMG (Fig. 1). Produjo en torno a quince coladas de lavas de las que apenas existe información petrológica ni geoquímica (una única muestra de afinidad calcoalcalina descrita en Hasenaka \& Carmichael, 1987). La edad de este volcán tampoco está bien definida, ya que solo existe una determinación radiométrica, basada en ${ }^{14} \mathrm{C}$, de $4700 \pm 200$ años (Hasenaka \& Carmichael, 1985).

\section{Métodos analíticos}

Los análisis de elementos mayores en roca total se realizaron con un espectrómetro secuencial de rayos $\mathrm{X}$ Siemens SRS 3000 siguiendo el procedimiento descrito en Lozano-Santa Cruz \& Bernal (2005) en el Laboratorio Universitario de Geoquímica Isotópica (LUGIS) del Instituto de Geología, Universidad Nacional Autónoma de México (UNAM).

La química mineral fue determinada mediante microsonda electrónica. Se utilizó una microsonda JEOL ${ }^{\circledR}$ "Superprobe JXA-8900 M" con cuatro analizadores WDS, del Centro Nacional de Microscopía Electrónica de la Universidad Complutense de
Madrid. Los análisis se realizaron sobre láminas pulidas metalizadas con una película de grafito y las condiciones de medida fueron de $15 \mathrm{kV}$ de diferencia de potencial y un haz de electrones de $20 \mathrm{nA}$ de intensidad de corriente y $5 \mu \mathrm{m}$ de diámetro. Los tiempos de medida fueron de 10 segundos en la posición del pico y 5 en la de cada fondo, con unos límites de detección de $0.5-6 \%$ en peso para óxidos con concentraciones $>1.5 \%$ en peso y $<10 \%$ para óxidos con concentraciones $<1.5 \%$ en peso. Los patrones usados fueron: sillimanita, albita, almandino, kaersutita, microclina, ilmenita, fluorapatito, escapolita, aleación de Ni, cromita, gahnita, bentonita y estroncianita que proceden del Smithsonian Institute (Washington DC, EEUU) y de la Universidad de Harvard (Boston, EEUU). Se analizaron $\mathrm{Si}, \mathrm{Al}, \mathrm{Fe}, \mathrm{Mn}, \mathrm{Mg}, \mathrm{Ca}, \mathrm{Na}, \mathrm{K}, \mathrm{Ti}, \mathrm{Ni}, \mathrm{Cr}$ y $\mathrm{P}$ en olivino, plagioclasa, clinopiroxeno, ortopiroxeno y anfíbol. Excepto en el caso del anfíbol, los análisis se realizaron exclusivamente en aquellos cristales o zonas de los cristales que no mostraban evidencias de desequilibrio respecto a la matriz que los engloba.

\section{Petrología y geoquímica}

En la Tabla 1 se recoge la composición en elementos mayores de las rocas objeto de estudio y en las Tablas 2 a 6, se resume la composición de las fases consideradas en cada una de dichas muestras.

Todas las rocas consideradas presentan textura hipocristalina, inequigranular, porfídica y vacuolar (Fig. 2). La matriz es microcristalina y está compuesta por plagioclasa (P1), ortopiroxeno (Opx), clinopiroxeno (Cpx), óxidos, olivino (O1), 
Tabla 1-Análisis químicos de roca total (\% en peso) de lavas del volcán El Metate

\begin{tabular}{lrrrrrrrrrrr}
\hline & M20 & M57 & M60 & M61 & M63 & M64 & M65 & M66 & M70 & M74 & M77 \\
\hline $\mathrm{SiO}_{2}$ & 61.29 & 55.02 & 57.90 & 60.07 & 59.40 & 60.09 & 57.02 & 59.26 & 58.11 & 60.23 & 60.69 \\
$\mathrm{TiO}_{2}$ & 0.67 & 0.92 & 0.80 & 0.69 & 0.70 & 0.68 & 0.90 & 0.68 & 0.73 & 0.69 & 0.60 \\
$\mathrm{Al}_{2} \mathrm{O}_{3}$ & 17.69 & 17.48 & 18.27 & 18.00 & 17.36 & 17.42 & 17.29 & 18.08 & 17.53 & 17.83 & 18.60 \\
$\mathrm{Fe}_{2} \mathbf{O}_{3}{ }^{\mathrm{t}}$ & 5.38 & 7.63 & 6.11 & 5.66 & 6.21 & 5.89 & 7.30 & 5.71 & 6.67 & 5.81 & 5.45 \\
$\mathrm{MnO}$ & 0.09 & 0.13 & 0.11 & 0.09 & 0.11 & 0.10 & 0.13 & 0.10 & 0.10 & 0.09 & 0.08 \\
$\mathbf{M g O}$ & 3.09 & 5.78 & 3.36 & 3.19 & 4.01 & 3.40 & 5.05 & 3.17 & 4.65 & 3.39 & 3.13 \\
$\mathrm{CaO}$ & 6.59 & 7.56 & 7.10 & 6.58 & 6.43 & 6.11 & 6.84 & 6.75 & 6.94 & 6.06 & 5.94 \\
$\mathrm{Na}_{2} \mathbf{O}$ & 3.91 & 3.44 & 3.94 & 3.98 & 3.68 & 3.78 & 3.63 & 4.03 & 3.73 & 3.93 & 3.99 \\
$\mathbf{K}_{2} \mathrm{O}$ & 1.45 & 1.18 & 1.61 & 1.48 & 1.80 & 1.76 & 1.49 & 1.41 & 1.52 & 1.89 & 1.43 \\
$\mathbf{P}_{2} \mathrm{O}_{5}$ & 0.21 & 0.05 & 0.11 & 0.00 & 0.02 & 0.01 & 0.05 & 0.04 & 0.21 & 0.27 & 0.17 \\
LOI & 0.34 & 0.50 & 0.26 & 0.00 & 0.24 & 0.25 & 0.00 & 0.30 & 0.22 & 0.25 & 0.52 \\
Total & 100.70 & 99.68 & 99.56 & 99.73 & 99.95 & 99.47 & 99.70 & 99.53 & 100.42 & 100.45 & 100.61 \\
\hline
\end{tabular}

*LOI: pérdida por calcinación.

Tabla 2-Análisis químicos, porcentaje de componente forsterita y temperatura calculada de fenocristales y microfenocristales de olivinos en lavas del volcán EI Metate

\begin{tabular}{|c|c|c|c|c|c|c|c|}
\hline & \multicolumn{6}{|c|}{ M57 } & \multirow{2}{*}{$\begin{array}{c}\text { M65 } \\
1\end{array}$} \\
\hline & 1 & 2 & 3 & 4 & 5 & 6 & \\
\hline & Feno. & Microfeno. & Feno. & Microfeno. & Feno. & Feno. & Feno. \\
\hline $\mathrm{SiO}_{2}$ & 38.96 & 38.92 & 39.07 & 39.94 & 36.84 & 37.99 & 37.42 \\
\hline $\mathrm{Al}_{2} \mathrm{O}_{3}$ & - & - & - & 0.01 & 0.22 & 0.04 & - \\
\hline $\mathrm{FeO}^{\top}$ & 19.68 & 21.14 & 20.79 & 15.99 & 23.45 & 22.68 & 25.24 \\
\hline MnO & 0.18 & 0.24 & 0.26 & 0.22 & 0.42 & 0.26 & 0.30 \\
\hline MgO & 41.28 & 40.78 & 40.15 & 44.89 & 36.29 & 38.16 & 36.24 \\
\hline $\mathrm{CaO}$ & 0.16 & 0.23 & 0.18 & 0.14 & 0.21 & 0.14 & 0.19 \\
\hline $\mathrm{Na}_{2} \mathrm{O}$ & 0.01 & - & - & 0.01 & 0.01 & 0.03 & 0.03 \\
\hline $\mathrm{K}_{2} \mathrm{O}$ & - & 0.00 & 0.01 & 0.01 & 0.01 & 0.01 & 0.02 \\
\hline $\mathrm{TiO}_{2}$ & 0.03 & 0.04 & 0.03 & 0.00 & 0.04 & - & 0.01 \\
\hline $\mathrm{NiO}$ & 0.08 & 0.07 & 0.20 & 0.27 & 0.07 & 0.09 & 0.12 \\
\hline $\mathrm{Cr}_{2} \mathrm{O}_{3}$ & 0.02 & - & - & - & - & - & 0.02 \\
\hline $\mathrm{P}_{2} \mathrm{O}_{5}$ & 0.05 & 0.09 & 0.05 & 0.04 & - & 0.03 & 0.04 \\
\hline $\mathbf{F}$ & - & 0.01 & - & - & - & - & - \\
\hline $\mathrm{Cl}$ & - & 0.01 & - & 0.00 & 0.03 & - & - \\
\hline Total & 100.44 & 101.52 & 100.73 & 101.51 & 97.55 & 99.42 & 99.62 \\
\hline$\%$ Fo & 79 & 77 & 77 & 83 & 73 & 75 & 72 \\
\hline $\mathrm{T}\left({ }^{\circ} \mathrm{C}\right)$ & 1212 & 1216 & 1218 & 1198 & 1232 & 1225 & 1225 \\
\hline
\end{tabular}

Análisis químicos expresados en \% en peso del óxido.

Todos los análisis en fenocristales han sido realizados en los bordes.

y ocasionalmente vidrio. A excepción de los óxi- El anfíbol (Amp) también aparece como fenocrisdos, las fases que componen la matriz aparecen tal en algunas de las muestras estudiadas. Por lo asimismo como feno y microfenocristales, siendo que respecta a su composición química (Tabla 1), $\mathrm{Pl}$ y piroxenos comunes a todas las muestras, las lavas de El Metate presentan un rango de variamientras que el $\mathrm{Ol}$ aparece solo ocasionalmente. ción en $\mathrm{SiO}_{2}$ relativamente reducido por lo que 
Tabla 3-Análisis químicos, porcentaje de los componentes An y Ab, y valores de temperatura ( $\mathrm{T}$ ), presión (P) y contenido en agua $\left(\mathrm{H}_{2} \mathrm{O} \%\right)$ calculados para cristales de plagioclasa en lavas del volcán El Metate

\begin{tabular}{|c|c|c|c|c|c|c|c|c|c|c|c|c|}
\hline & M20 & M57 & M60 & M61 & M63 & M & & M65 & & M6e & & M77 \\
\hline & 1 & 1 & 1 & 1 & 1 & 1 & 2 & 1 & 1 & 2 & 3 & 1 \\
\hline & Feno. & Microfeno. & Feno. & Feno. & Matriz & Feno. & Matriz & Matriz & Feno. & Feno. & Microfeno. & Matriz \\
\hline $\mathrm{SiO}_{2}$ & 53.03 & 53.58 & 52.51 & 52.90 & 49.91 & 54.71 & 52.18 & 50.54 & 51.87 & 52.87 & 52.45 & 54.14 \\
\hline $\mathrm{Al}_{2} \mathrm{O}_{3}$ & 28.22 & 29.05 & 29.01 & 28.35 & 30.59 & 27.84 & 28.42 & 30.72 & 28.96 & 28.02 & 28.62 & 28.98 \\
\hline $\mathrm{FeO}^{\top}$ & 0.68 & 0.76 & 0.66 & 0.93 & 0.71 & 0.86 & 0.93 & 0.61 & 0.75 & 0.85 & 0.57 & 0.69 \\
\hline MnO & - & - & 0.02 & - & 0.03 & - & 0.05 & 0.03 & 0.04 & 0.09 & - & - \\
\hline MgO & 0.10 & 0.17 & 0.06 & 0.05 & 0.06 & 0.05 & - & 0.09 & 0.12 & 0.08 & 0.08 & 0.08 \\
\hline $\mathrm{CaO}$ & 12.17 & 12.21 & 12.60 & 11.42 & 14.90 & 11.19 & 12.78 & 14.54 & 12.84 & 12.34 & 12.87 & 12.02 \\
\hline $\mathrm{Na}_{2} \mathrm{O}$ & 4.49 & 3.88 & 4.70 & 4.93 & 3.43 & 5.17 & 4.53 & 3.31 & 4.37 & 4.81 & 4.65 & 4.56 \\
\hline $\mathrm{K}_{2} \mathrm{O}$ & 0.15 & 0.17 & 0.18 & 0.07 & 0.15 & 0.36 & 0.30 & 0.15 & 0.13 & 0.11 & 0.11 & 0.13 \\
\hline $\mathrm{TiO}_{2}$ & 0.01 & 0.01 & 0.01 & 0.06 & 0.02 & 0.01 & 0.07 & 0.01 & 0.01 & 0.01 & - & 0.07 \\
\hline $\mathrm{NiO}$ & 0.08 & - & 0.05 & 0.02 & 0.03 & - & - & 0.02 & 0.02 & - & 0.03 & - \\
\hline $\mathrm{Cr}_{2} \mathrm{O}_{3}$ & 0.01 & - & 0.00 & 0.00 & - & 0.03 & - & - & 0.00 & - & - & - \\
\hline $\mathbf{P}_{2} \mathbf{O}_{5}$ & 0.08 & 0.02 & 0.02 & 0.07 & 0.00 & - & 0.04 & - & 0.00 & 0.03 & 0.07 & 0.01 \\
\hline $\mathbf{F}$ & 0.03 & - & 0.05 & 0.01 & 0.06 & 0.06 & - & 0.03 & 0.00 & - & - & 0.02 \\
\hline $\mathrm{Cl}$ & 0.01 & 0.00 & 0.00 & 0.03 & 0.00 & 0.02 & 0.01 & 0.00 & 0.03 & 0.00 & - & 0.00 \\
\hline Total & 99.05 & 99.84 & 99.87 & 98.83 & 99.87 & 100.29 & 99.30 & 100.06 & 99.12 & 99.21 & 99.46 & 100.70 \\
\hline$\%$ An & 60.0 & 63.5 & 59.7 & 56.1 & 70.6 & 54.5 & 60.9 & 70.8 & 61.9 & 58.6 & 60.5 & 59.3 \\
\hline$\% \mathrm{Ab}$ & 40.0 & 36.5 & 40.3 & 43.9 & 29.4 & 45.5 & 39.1 & 29.2 & 38.1 & 41.4 & 39.5 & 40.7 \\
\hline $\mathrm{T}\left({ }^{\circ} \mathrm{C}\right)$ & 1135 & 1148 & 1150 & 1148 & 1144 & 1126 & 1133 & 1162 & 1147 & 1143 & 1145 & 1128 \\
\hline $\mathrm{H}_{2} \mathrm{O} \%$ & 1.7 & 1.5 & 1.5 & 1.5 & 1.5 & 1.8 & 1.6 & 1.2 & 1.6 & 1.7 & 1.6 & 2.1 \\
\hline P (Kbar) & 5.4 & 4.7 & 7.3 & 7.2 & 3.7 & 6.5 & 5.3 & 3.6 & 5.9 & 6.5 & 6.1 & 5.2 \\
\hline
\end{tabular}

Análisis químicos expresados en \% en peso del óxido. Todos los análisis en fenocristales han sido realizados en los bordes.

T calculada según ecuación 3; H2O calculada según ecuación 4; P calculada según ecuación 5 .

están representadas casi exclusivamente por términos andesíticos (Fig. 3), con bajas relaciones $\mathrm{FeO}^{\mathrm{T}} / \mathrm{MgO}$. Los relativamente altos contenidos de $\mathrm{SiO}_{2}(61-55 \%)$ y bajos de $\mathrm{MgO}(5.8-3 \%)$ y del índice \#Mg (66-58) indican que en todos los casos se trata de rocas evolucionadas.

En general las concentraciones de $\mathrm{MgO}, \mathrm{Fe}_{2} \mathrm{O}_{3}$, y $\mathrm{CaO}$, disminuyen respecto a $\mathrm{SiO}_{2}$ (Fig. 4), mientras que $\mathrm{K}_{2} \mathrm{O}, \mathrm{Na}_{2} \mathrm{O}, \mathrm{P}_{2} \mathrm{O}_{5}$ y en cierta medida $\mathrm{Al}_{2} \mathrm{O}_{3}$, tienden a aumentar. Sin embargo, la distribución de estas rocas en los diagramas $\mathrm{SiO}_{2}^{-}$ elemento revela la existencia de dos tendencias contrastadas. Como se puede observar sobre todo en el diagrama $\mathrm{SiO}_{2}-\mathrm{MgO}$, un grupo de muestras (M-20, M-60, M-61 y M-66), se distribuyen a lo largo de una pauta de menor pendiente respecto a la trayectoria representada por el resto. Asimismo las pautas generales de enriquecimiento/empobrecimiento no son siempre coincidentes. Así el primer grupo de muestras exhibe un empobrecimiento en $\mathrm{K}_{2} \mathrm{O}, \mathrm{Na}_{2} \mathrm{O}$ y $\mathrm{Al}_{2} \mathrm{O}_{3}$ al aumentar el contenido de $\mathrm{SiO}_{2}$, mientras que el resto muestra un enriquecimiento.

\section{Fases minerales}

\section{Olivino}

Los olivinos son en su mayoría feno y microfenocristales (Fig. 2A), aunque también aparecen como microcristales formando la matriz. En general son subidiomorfos, de bordes rotos o con golfos de corrosión, así como con zonas internas huecas. Si bien al microscopio es difícil de distinguir, en la microsonda se ha podido detectar que los fenocristales pueden presentar bordes de recrecimiento y en algún caso, una fina corona de Opx. El olivino aparece exclusivamente en las muestras con mayor 
Tabla 4-Análisis químicos, número de magnesio y valores de temperatura $(\mathrm{T})$ y presión $(\mathrm{P})$ calculados para cristales de ortopiroxeno en lavas del volcán El Metate

\begin{tabular}{|c|c|c|c|c|c|c|c|c|c|c|}
\hline & \multicolumn{2}{|c|}{ M60 } & \multicolumn{2}{|c|}{ M64 } & \multirow{2}{*}{$\begin{array}{c}\text { M65 } \\
1\end{array}$} & \multicolumn{3}{|c|}{ M66 } & \multirow{2}{*}{$\begin{array}{c}\text { M70 } \\
1\end{array}$} & \multirow{2}{*}{$\begin{array}{c}\text { M74 } \\
1\end{array}$} \\
\hline & 1 & 2 & 1 & 2 & & 1 & 2 & 3 & & \\
\hline & Microfeno. & Microfeno. & Feno. & Microfeno. & Matriz & Matriz & Microfeno. & Microfeno. & Microfeno. & Microfeno. \\
\hline $\mathrm{SiO}_{2}$ & 55.30 & 55.11 & 52.95 & 52.65 & 54.22 & 53.69 & 53.13 & 54.49 & 53.91 & 55.74 \\
\hline $\mathrm{Al}_{2} \mathrm{O}_{3}$ & 1.22 & 0.93 & 0.80 & 1.96 & 0.24 & 1.58 & 2.19 & 0.92 & 0.46 & 0.83 \\
\hline $\mathrm{FeO}^{\mathrm{T}}$ & 12.33 & 12.59 & 16.92 & 15.50 & 14.89 & 13.39 & 13.73 & 12.70 & 15.00 & 12.95 \\
\hline MnO & 0.36 & 0.38 & 0.45 & 0.35 & 0.28 & 0.38 & 0.23 & 0.28 & 0.32 & 0.23 \\
\hline $\mathrm{MgO}$ & 28.40 & 28.93 & 26.44 & 27.10 & 27.06 & 28.40 & 27.98 & 28.61 & 27.38 & 29.36 \\
\hline $\mathrm{CaO}$ & 1.42 & 1.55 & 1.68 & 1.76 & 2.18 & 2.14 & 1.62 & 1.60 & 2.05 & 1.59 \\
\hline $\mathrm{Na}_{2} \mathrm{O}$ & 0.05 & 0.05 & 0.02 & 0.03 & 0.05 & 0.05 & 0.02 & 0.04 & 0.04 & 0.04 \\
\hline $\mathrm{K}_{2} \mathrm{O}$ & 0.01 & 0.00 & 0.00 & 0.01 & 0.01 & 0.03 & - & - & 0.00 & 0.00 \\
\hline $\mathrm{TiO}_{2}$ & 0.28 & 0.30 & 0.21 & 0.35 & 0.19 & 0.32 & 0.31 & 0.16 & 0.21 & 0.07 \\
\hline $\mathrm{NiO}$ & 0.02 & 0.01 & 0.03 & 0.00 & 0.00 & 0.02 & 0.01 & 0.06 & 0.00 & 0.00 \\
\hline $\mathrm{Cr}_{2} \mathrm{O}_{3}$ & 0.00 & 0.00 & 0.00 & 0.03 & 0.01 & 0.01 & 0.02 & - & 0.00 & 0.02 \\
\hline $\mathrm{P}_{2} \mathrm{O}_{5}$ & 0.01 & 0.07 & 0.00 & 0.00 & 0.00 & 0.06 & 0.02 & 0.01 & 0.03 & 0.00 \\
\hline $\mathbf{F}$ & 0.04 & 0.00 & 0.00 & 0.02 & 0.02 & 0.00 & - & - & 0.00 & 0.00 \\
\hline $\mathrm{Cl}$ & 0.01 & 0.02 & 0.00 & 0.02 & 0.00 & 0.00 & 0.02 & - & 0.00 & 0.01 \\
\hline Total & 99.42 & 99.92 & 99.50 & 99.77 & 99.15 & 100.08 & 99.24 & 98.86 & 99.39 & 100.85 \\
\hline \#Mg & 80 & 81 & 78 & 80 & 79 & 74 & 81 & 81 & 79 & 73 \\
\hline $\mathrm{T}\left({ }^{\circ} \mathrm{C}\right)$ & 1078 & 1147 & 1027 & 1042 & 1092 & 1053 & 1104 & 1113 & 1071 & 1066 \\
\hline P (Kbar) & 2.1 & 2.2 & 0.0 & 0.2 & 2.8 & 0.6 & 0.1 & 1.1 & 2.0 & 0.7 \\
\hline
\end{tabular}

Análisis químicos expresados en \% en peso del óxido. Todos los análisis en fenocristales han sido realizados en los bordes T calculada según ecuación 6; $\mathrm{P}$ calculada según ecuación 7.

contenido en $\mathrm{MgO}(>4.5 \%)$ y muestran un rango composicional relativamente estrecho entre Fo83 y Fo72 (Tabla 2).

\section{Plagioclasa}

La plagioclasa se encuentra presente en todas las rocas estudiadas como fenocristales, microfenocristales, formando parte de la matriz y como inclusiones microcristalinas en fenocristales de otras fases. Es posible distinguir cuatro poblaciones principales de cristales de Pl (Fig. 2B, C y D):

Tipo 1: Cristales idio-subidiomorfos que pueden ser tanto feno como microfenocristales o cristales de la matriz y que presentan macla de Carlsbad o polisintética. Esporádicamente algunos fenocristales pueden tener extinción ondulante o presentar un núcleo alterado.

Tipo 2:Feno y microfenocristales con zonación oscilatoria muy marcada, en ocasiones con extinción ondulante sobreimpuesta, que pueden tener bordes corroídos y recrecimientos y/o núcleos alterados y con inclusiones. Con frecuencia en estas plagioclasas se encuentra gran cantidad de microcristales de apatito incluidos en los bordes recrecidos (que también se observan en la matriz en algunas muestras), siendo las inclusiones del núcleo indistinguibles.

Tipo 3: Fenocristales totalmente alterados y de bordes corroídos, en los que se sigue apreciando el maclado, y con un borde de recrecimiento también corroído (textura de reabsorción generalizada tipo "spongy cellular, sieved" (ver Streck, 2008).

Tipo 4: Microcristales que se encuentran en coronas que rodean a los cristales de anfíbol o como inclusiones en algunos piroxenos (Fig. 2D).

El rango composicional observado en las plagioclasas analizadas es relativamente amplio, entre An71 y An54, y corresponden en su mayor parte a labradoritas (Fig. 5), excepto dos ejemplares de bitownita identificados en la matriz microcristalina. A pesar de la existencia de cuatro poblaciones principales de plagioclasas, el rango 
Tabla 5-Análisis químicos, número de magnesio y valores de temperatura $(T)$ y presión $(P)$ calculados para cristales de clinopiroxeno en lavas del volcán EI Metate

\begin{tabular}{|c|c|c|c|c|c|c|c|c|c|c|c|c|}
\hline & \multicolumn{2}{|c|}{ M60 } & \multirow{2}{*}{$\begin{array}{c}\text { M61 } \\
1\end{array}$} & \multirow{2}{*}{$\begin{array}{c}\text { M63 } \\
1\end{array}$} & \multicolumn{3}{|c|}{ M64 } & \multirow{2}{*}{$\begin{array}{c}\text { M66 } \\
1\end{array}$} & \multicolumn{2}{|c|}{ M70 } & \multirow{2}{*}{$\begin{array}{c}\text { M74 } \\
1\end{array}$} & \multirow{2}{*}{$\begin{array}{c}\text { M77 } \\
1\end{array}$} \\
\hline & 1 & 2 & & & 1 & 2 & 3 & & 1 & 2 & & \\
\hline & Matriz & Microfeno. & Feno. & Feno. & Feno. & Microfeno. & Microfeno. & Feno. & Feno. & Feno. & Feno. & Feno. \\
\hline $\mathrm{SiO}_{2}$ & 51.36 & 49.18 & 51.12 & 49.80 & 50.48 & 50.313 & 51.941 & 50.64 & 50.20 & 50.79 & 49.91 & 50.90 \\
\hline $\mathrm{Al}_{2} \mathrm{O}_{3}$ & 2.85 & 3.89 & 3.32 & 3.50 & 3.01 & 2.706 & 2.302 & 2.81 & 3.66 & 2.98 & 3.45 & 2.69 \\
\hline $\mathrm{FeO}^{\top}$ & 7.61 & 8.15 & 6.36 & 8.23 & 6.70 & 9.051 & 6.587 & 9.31 & 8.01 & 8.89 & 8.05 & 7.91 \\
\hline MnO & 0.34 & 0.28 & 0.20 & 0.20 & 0.08 & 0.14 & 0.124 & 0.26 & 0.15 & 0.12 & 0.20 & 0.22 \\
\hline MgO & 16.44 & 15.13 & 16.42 & 15.14 & 16.52 & 15.465 & 16.599 & 18.42 & 14.75 & 16.14 & 15.55 & 15.51 \\
\hline $\mathrm{CaO}$ & 20.84 & 21.77 & 22.62 & 21.13 & 21.78 & 20.594 & 22.026 & 17.39 & 21.09 & 18.65 & 21.47 & 21.71 \\
\hline $\mathrm{Na}_{2} \mathrm{O}$ & 0.36 & 0.45 & 0.36 & 0.35 & 0.31 & 0.337 & 0.261 & 0.31 & 0.36 & 0.33 & 0.40 & 0.27 \\
\hline $\mathrm{K}_{2} \mathrm{O}$ & 0.06 & - & 0.01 & 0.03 & 0.00 & - & 0.012 & 0.00 & 0.00 & 0.03 & - & 0.00 \\
\hline $\mathrm{TiO}_{2}$ & 0.55 & 1.00 & 0.41 & 0.74 & 0.50 & 0.674 & 0.342 & 0.72 & 0.74 & 0.79 & 0.50 & 0.45 \\
\hline $\mathrm{NiO}$ & - & 0.05 & 0.05 & 0.10 & 0.02 & 0.034 & 0.034 & - & 0.04 & - & 0.02 & 0.03 \\
\hline $\mathrm{Cr}_{2} \mathrm{O}_{3}$ & - & 0.07 & 0.06 & 0.07 & 0.29 & - & 0.094 & 0.03 & 0.07 & - & 0.03 & - \\
\hline $\mathrm{P}_{2} \mathrm{O}_{5}$ & 0.00 & 0.29 & 0.03 & 0.01 & 0.04 & - & 0.016 & - & 0.08 & 0.01 & 0.05 & 0.03 \\
\hline $\mathbf{F}$ & - & 0.05 & 0.05 & - & 0.07 & - & 0.023 & 0.00 & 0.02 & - & 0.01 & 0.06 \\
\hline $\mathrm{Cl}$ & 0.02 & 0.01 & - & 0.03 & 0.00 & - & - & 0.02 & 0.01 & - & 0.00 & - \\
\hline Total & 100.43 & 100.31 & 101.00 & 99.33 & 99.79 & 99.31 & 100.36 & 99.90 & 99.17 & 98.71 & 99.62 & 99.76 \\
\hline \#Mg & 89 & 92 & 97 & 87 & 96 & 84 & 91 & 89 & 83 & 80 & 91 & 88 \\
\hline $\mathrm{T}\left({ }^{\circ} \mathrm{C}\right)$ & 1111 & 1098 & 1069 & 1097 & 1045 & 1080 & 1069 & 1018 & 1128 & 1147 & 1099 & 1064 \\
\hline P (Kbar) & 4.9 & 5.9 & 3.6 & 5.5 & 2.5 & 4.1 & 2.9 & 0.4 & 6.3 & 6.5 & 6.3 & 3.6 \\
\hline
\end{tabular}

Análisis químicos expresados en \% en peso del óxido. Todos los análisis en fenocristales han sido realizados en los bordes.

T calculada según ecuación 9; $P$ calculada según ecuación 8.

composicional observado en los análisis de feno- y microfenocristales y microcristales (ver la sección de geotermometría) muestran un rango equivalente al del conjunto de plagioclasas, con cierta tendencia de los cristales de la matriz a proyectarse en el extremo más anortítico del espectro (labradorita-bitownita), mientras que los bordes de microfenocristales se proyectan en la región de tendencia albítica.

Las plagioclasas de Tipo 3 (de textura de reabsorción generalizada tipo "spongy cellular sieved") no han podido ser caracterizadas geoquímicamente dado el alto grado de alteración que presentan. Tampoco se han caracterizado las plagioclasas presentes en las coronas de los anfíboles, ya que pueden representar productos de reacciones de desequilibrio.

\section{Ortopiroxeno}

El ortopiroxeno raramente se encuentra como fenocristal, pero es frecuente como microfenocristal, asi como en la matriz o formando parte del núcleo de un cristal con recrecimiento de clinopiroxeno.
Los cristales analizados se clasifican como enstatitas (Fig. 5) y tienen un rango composicional que oscila entre valores de $\# \mathrm{Mg}\left(\# \mathrm{Mg}=\mathrm{Mg} /\left(\mathrm{Mg}+\mathrm{Fe}^{+2}\right) \times 100\right)$ desde $81 \%$ a $73 \%$ (Tabla 4) para los microfenocristales, mientras que los cristales de la matriz se proyectan en un rango composicional algo más estrecho (79-74\%).

\section{Clinopiroxeno}

El clinopiroxeno aparece como fenocristales, microfenocristales así como en la matriz. La mayor parte de los microfeno y microcristales tienen inclusiones de opacos, mientras que los fenocristales muestran zonas isótropas, principalmente en el núcleo, y se caracterizan por tener un estrecho borde de recrecimiento (Fig. 2A). Algunos cristales exhiben además características particulares: pueden encontrarse en continuidad óptica con Opx formando un zonado en parches, o bien hallarse en forma de agregados de cristales con zonación concéntrica, donde el núcleo está formado por Opx y las zonas externas por Cpx.

$\mathrm{El}$ valor \#Mg de los $\mathrm{Cpx}$ analizados varía entre $97-80 \%$ (Tabla 5) y se proyectan como augitas en 
Tabla 6-Análisis químicos, clasificación y valores de temperatura $(T)$, presión $(P)$ y contenido en agua del fundico $\left(\mathrm{H}_{2} \mathrm{O}\right)$ calculados para cristales de anfíbol en lavas del volcán EI Metate

\begin{tabular}{|c|c|c|c|c|c|c|c|c|c|c|c|}
\hline & \multicolumn{2}{|c|}{ M20 } & \multirow{2}{*}{\multicolumn{3}{|c|}{$\begin{array}{c}\text { M60 } \\
1\end{array}$}} & \multirow{3}{*}{$\begin{array}{c}\text { M61 } \\
1 \\
\text { Centro }\end{array}$} & \multicolumn{2}{|c|}{ M66 } & \multirow{2}{*}{\multicolumn{2}{|c|}{$\begin{array}{c}\text { M74 } \\
1\end{array}$}} & \multirow{3}{*}{$\begin{array}{c}\text { M77 } \\
1 \\
\text { Centro }\end{array}$} \\
\hline & \multirow{2}{*}{$\begin{array}{c}1 \\
\text { Medio }\end{array}$} & \multirow{2}{*}{$\begin{array}{c}2 \\
\text { Centro }\end{array}$} & & & & & 1 & 2 & & & \\
\hline & & & a Centro & b Medio & c Borde & & Centro & Centro & a Centro & b Borde & \\
\hline $\mathrm{SiO}_{2}$ & 45.04 & 45.13 & 42.96 & 41.72 & 42.57 & 43.69 & 42.58 & 43.68 & 43.54 & 44.23 & 44.839 \\
\hline $\mathrm{Al}_{2} \mathrm{O}_{3}$ & 11.38 & 11.19 & 12.51 & 12.90 & 12.66 & 12.72 & 11.97 & 10.74 & 12.19 & 11.25 & 11.001 \\
\hline $\mathrm{FeO}^{\top}$ & 8.69 & 8.16 & 8.37 & 10.73 & 9.64 & 9.41 & 10.14 & 10.33 & 11.11 & 8.53 & 8.349 \\
\hline MnO & 0.08 & 0.11 & 0.04 & 0.09 & 0.09 & 0.12 & 0.14 & 0.14 & 0.12 & 0.08 & 0.161 \\
\hline MgO & 16.45 & 17.57 & 16.74 & 14.99 & 16.37 & 15.47 & 15.88 & 15.64 & 15.02 & 16.57 & 16.697 \\
\hline $\mathrm{CaO}$ & 12.17 & 11.99 & 12.28 & 11.54 & 11.74 & 11.68 & 11.95 & 11.79 & 11.82 & 11.85 & 11.159 \\
\hline $\mathrm{Na}_{2} \mathrm{O}$ & 2.18 & 2.09 & 2.40 & 2.36 & 2.55 & 2.35 & 2.38 & 2.39 & 2.25 & 2.23 & 2.152 \\
\hline $\mathrm{K}_{2} \mathrm{O}$ & 0.38 & 0.37 & 0.54 & 0.51 & 0.51 & 0.49 & 0.39 & 0.37 & 0.52 & 0.61 & 0.382 \\
\hline $\mathrm{TiO}_{2}$ & 1.94 & 1.55 & 2.08 & 2.35 & 1.97 & 1.50 & 2.13 & 2.30 & 1.38 & 1.38 & 1.204 \\
\hline $\mathrm{NiO}$ & - & 0.10 & 0.05 & - & 0.13 & - & 0.12 & - & 0.04 & 0.03 & 0.008 \\
\hline $\mathrm{Cr}_{2} \mathrm{O}_{3}$ & 0.07 & 0.34 & 0.29 & 0.01 & 0.02 & - & 0.01 & 0.03 & 0.04 & 0.13 & 0.095 \\
\hline $\mathrm{P}_{2} \mathrm{O}_{5}$ & - & - & 0.02 & - & 0.02 & 0.03 & 0.03 & 0.02 & 0.02 & 0.03 & 0.044 \\
\hline$F$ & 0.11 & 0.02 & 0.12 & 0.09 & 0.15 & 0.06 & 0.11 & 0.02 & 0.06 & 0.08 & 0.051 \\
\hline Cl & 0.04 & 0.04 & 0.03 & 0.04 & 0.04 & 0.04 & 0.10 & 0.02 & 0.04 & 0.03 & 0.01 \\
\hline Total & 98.52 & 98.64 & 98.41 & 97.32 & 98.44 & 97.54 & 97.93 & 97.47 & 98.14 & 97.03 & 96.15 \\
\hline Clasificación & $\begin{array}{l}\text { magnesio- } \\
\text { Hastingsita }\end{array}$ & $\begin{array}{l}\text { magnesio- } \\
\text { Hastingsita }\end{array}$ & $\begin{array}{l}\text { magnesio- } \\
\text { Hastingsita }\end{array}$ & $\begin{array}{l}\text { magnesio- } \\
\text { Hastingsita }\end{array}$ & $\begin{array}{l}\text { magnesio- } \\
\text { Hastingsita }\end{array}$ & Pargasita & $\begin{array}{l}\text { magnesio- } \\
\text { Hastingsita }\end{array}$ & $\begin{array}{l}\text { magnesio- } \\
\text { Hastingsita }\end{array}$ & $\begin{array}{l}\text { magnesio- } \\
\text { Hastingsita }\end{array}$ & $\begin{array}{l}\text { magnesio- } \\
\text { Hastingsita }\end{array}$ & $\begin{array}{l}\text { magnesio- } \\
\text { Hastingsita }\end{array}$ \\
\hline $\mathrm{T}\left({ }^{\circ} \mathrm{C}\right)$ & 944 & 943 & 995 & 990 & 990 & 964 & 978 & 922 & 954 & 946 & 943 \\
\hline$P$ (Kbar) & 2.9 & 2.7 & 3.9 & 4.5 & 4.0 & 4.2 & 3.5 & 2.8 & 3.7 & 3.0 & 2.7 \\
\hline $\mathrm{H}_{2} \mathrm{O}(\%)$ & 6.0 & 5.5 & 5.5 & 6.3 & 5.5 & 6.9 & 5.7 & 5.2 & 6.5 & 5.3 & 5.9 \\
\hline
\end{tabular}

Análisis químicos expresados en \% en peso del óxido.

T calculada según ecuación 10; $\mathrm{P}$ calculada según ecuación 11; $\mathrm{H}_{2} \mathrm{O}$ calculada según ecuación 12. 

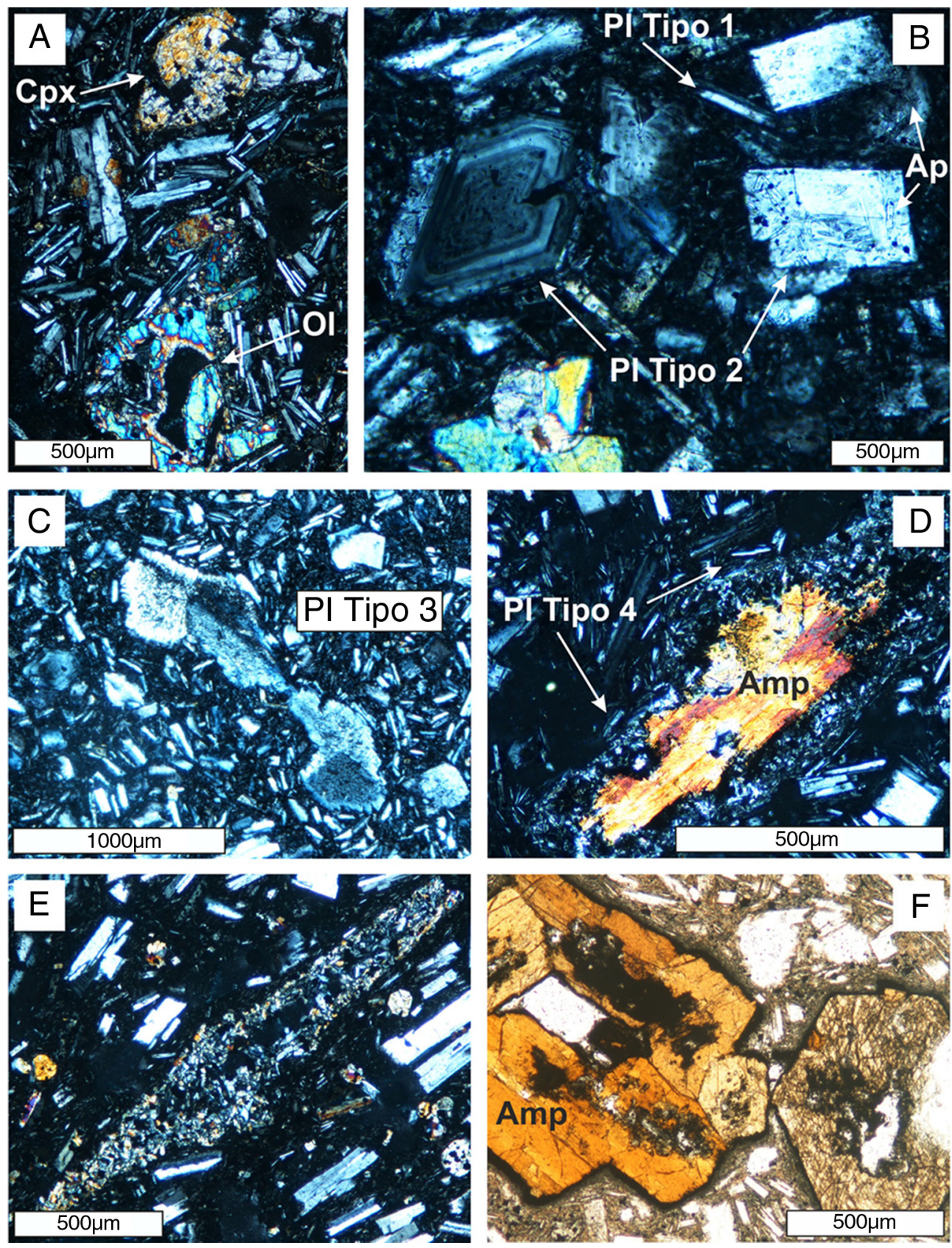

Fig. 2.-A) Fenocristales de olivino y clinopiroxeno en una matriz de plagioclasas de Tipo 4 (muestra M-57, nícoles cruzados). B-C) Ejemplos de plagioclasas de los tipos 1, 2 y 3 e inclusiones de apatito (muestra M-64, nícoles cruzados); D) Anfíbol con corona de opacita granular compuesta por Plg+Cpx+óxidos (muestra M-20, nícoles cruzados); E) pseudomorfo de anfíbol completamente reemplazado (muestra M-60, nícoles cruzados); F) Anfíboles con corona de opacita simplectítica (muestra M-57, nícoles paralelos).

el diagrama de clasificación Mg-Ca-Fe (Fig. 5). Los cristales de la matriz y los microfenocristales presentan las composiciones más magnésicas, con rangos de \#Mg entre 84-92\%. En cambio, los bordes de los fenocristales analizados abarcan un amplio abanico de composiciones ( $\# \mathrm{Mg}=80-97 \%$ ).
Anfíbol

El Amp aparece como fenocristal y también como microfenocristal y muestra una compleja historia de alteración que incluye dos tipos de opacitización (sensu Plechov et al., 2008). Por un lado, 


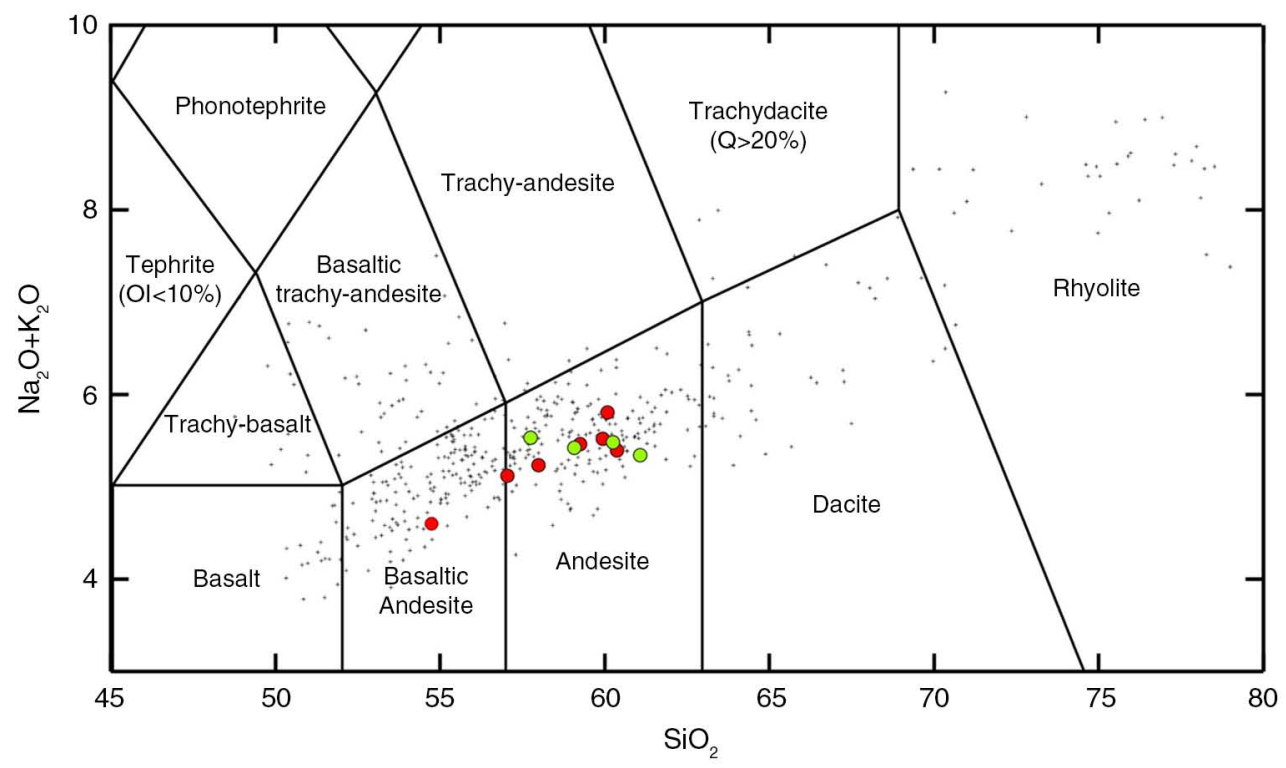

Fig. 3.-Proyección de las lavas de El Metate (círculos rojos y verdes) y rocas del Campo Volcánico de Michoacán-Guanajuato (cruces, según datos en Gómez-Tuena et al., 2007 y referencias incluidas) en el diagrama TAS (Le Bas et al., 1992). Datos en porcentaje en peso.
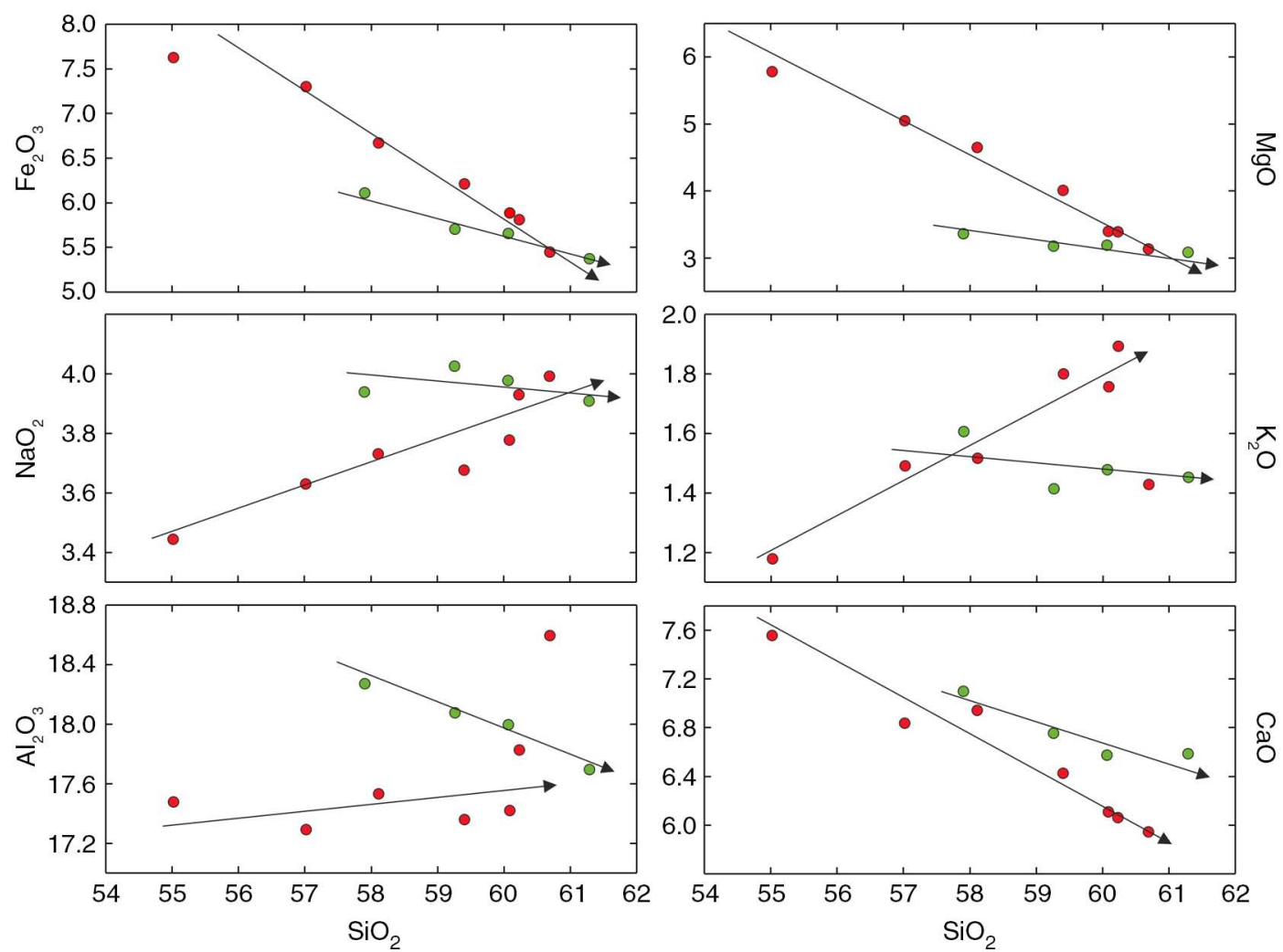

Fig. 4.-Diagramas $\mathrm{SiO}_{2}$-óxidos (en \% en peso) para las lavas de El Metate. Se indican las dos pautas de diferenciación observadas.

se puede observar opacitización en coronas, que a su vez están compuestas por dos zonas: una parte interna que se desarrolla directamente en contacto con el anfíbol (opacita granular) y compuesta por $\mathrm{Pl}$, Cpx y óxidos de entre 3 y $10 \mu \mathrm{m}$; y una parte externa de opacita simplectítica, que en ocasiones 

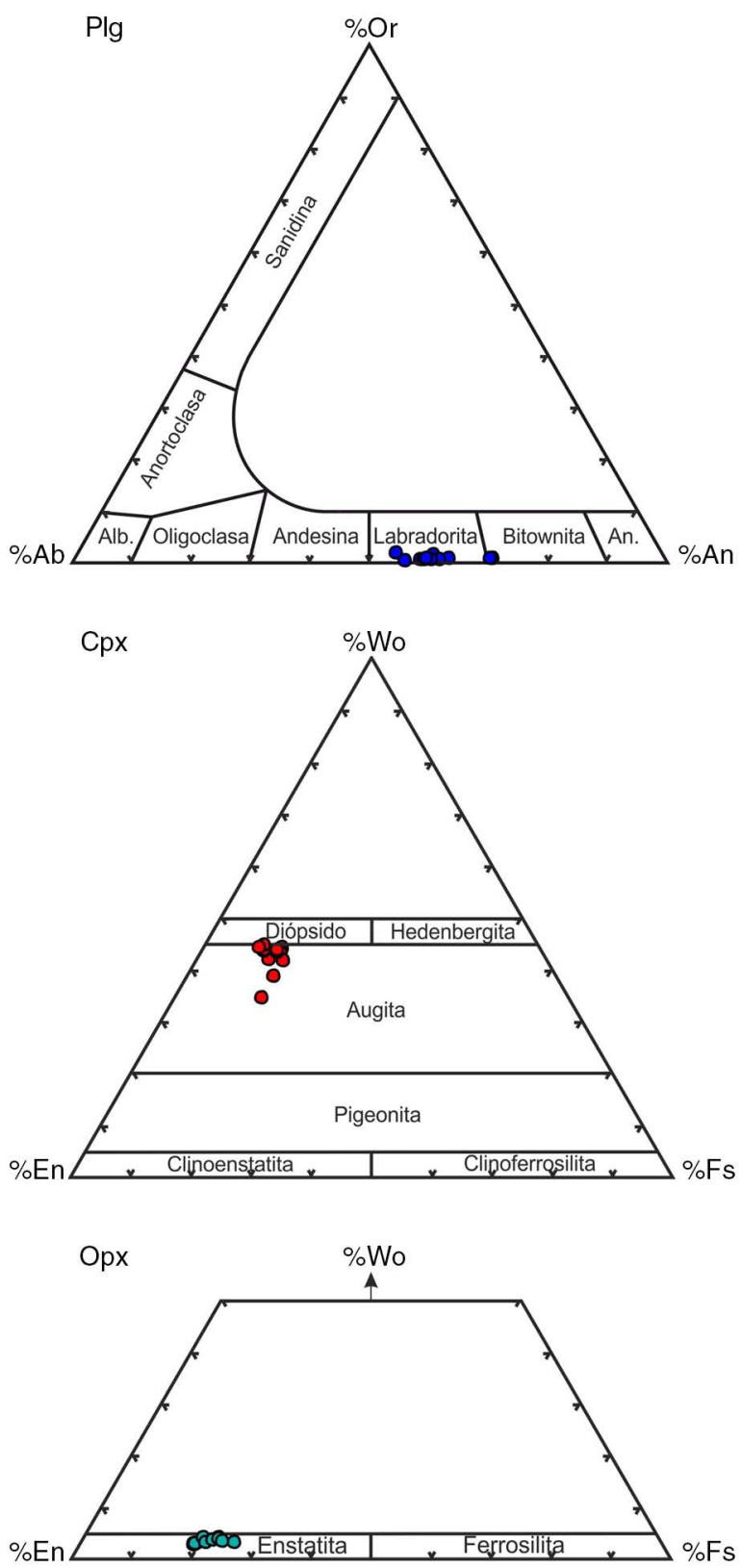

Fig. 5.-Diagramas de clasificación de plagioclasas (Ab-An-Or) y de piroxenos (Wo-En-Fe).

es la única que se desarrolla, compuesta por un agregado isótropo de minerales de grano fino cuya composición no pudo ser determinada. Por otro lado, también se observa opacitización en forma de áreas de descomposición volumétrica. Se denomina de este modo a zonas de reemplazamiento en el interior del cristal que han sido alteradas a través de planos de debilidad y que en este caso están compuestas mayoritariamente por cristales de clinopiroxeno que heredan la orientación estructural del anfibol al que reemplazan.

Atendiendo al tipo de corona de opacita que presentan, se pueden distinguir dos tipos de cristales: a) los casi euhedrales con tamaños que pueden ir desde fenocristales hasta microfenocristales, que no suelen presentar alteración en áreas de descomposición volumétrica y muestran únicamente coronas de opacita simplectítica (isótropa) (Fig. 2F), y b) los que presentan corona de opacita granular de $\mathrm{Pl}, \mathrm{Cpx}$ y opacos muy desarrollada (Fig. 2D), que en ocasiones llega a reemplazar todo el mineral (pseudomorfos) (Fig. 2E). Estos cristales no suelen presentar opacita isótropa y son de tamaño inferior a los primeros. Todas las muestras con anfíbol incluyen ambos tipos, si bien los primeros son los más frecuentes.

En todos los casos se corresponden a anfíboles cálcicos y se clasifican como Mg-hastingsitas, a excepción de una pargasita (mayor contenido en $\mathrm{Na}$ respecto a $\mathrm{Ca}$ ). No se han observado diferencias composicionales entre los diferentes tipos de cristales identificados petrográficamente.

\section{Geotermobarometría}

A partir de las diferentes formulaciones de geotermómetros y geobarómetros disponibles en la literatura (ver Putirka, 2008) se seleccionaron aquellos que mejor se adaptan en cada caso al rango composicional de las rocas objeto de estudio, así como a la paragénesis representada por $\mathrm{Pl}, \mathrm{Cpx}, \mathrm{Opx}, \mathrm{Ol}$ y Amp.

La mayoría de estos geotermobarómetros estiman las condiciones P-T a partir de equilibrios químicos mineral-mineral o mineral-líquido. En consecuencia, para aplicarlos de forma estricta, solo se pueden considerar aquellos cristales o zonas de los mismos, que no presenten evidencias de desestabilización y se encuentren en contacto con la fase (sea mineral o líquido) con la que se encuentra en equilibrio. En consecuencia, los cálculos basados en equilibrios mineral-líquido se han limitado a las composiciones obtenidas en aquellas zonas de los minerales sin texturas de desequilibrio y que se encuentran en contacto con la matriz, asumiendo que la composición de la roca total representa de forma aproximada la composición del magma en equilibrio con las fases que contiene. Este es el caso de los termobarómetros 
para $\mathrm{Ol}, \mathrm{Cpx}, \mathrm{Opx}$ y $\mathrm{Pl}$, que se basan en relaciones de intercambio entre el mineral y el fundido en equilibrio. Sin embargo, para el Amp las consideraciones anteriores no son aplicables debido a que el termobarómetro disponible utiliza únicamente la composición del mineral, por lo que se pueden utilizar tanto las composiciones obtenidas en el centro como en el borde del cristal.

\section{Olivino}

En el caso del olivino hemos adoptado la ecuación 22 de Putirka (2008), basada en el equilibrio olivino-líquido:

$$
\begin{aligned}
\mathrm{T}\left({ }^{\circ} \mathrm{C}\right)= & \left\{15294.6+1318.8 \mathrm{P}(\mathrm{GPa})+2.4834[\mathrm{P}(\mathrm{GPa})]^{2}\right\} / \\
& \left\{8.048+2.8352 \ln \mathrm{D}_{\mathrm{Mg}}^{\mathrm{O} / \mathrm{liq}}+2.097 \ln \left[1.5\left(\mathrm{C}_{\mathrm{NM}}^{\mathrm{L}}\right)\right]\right. \\
& +2.575 \ln \left[3\left(\mathrm{C}_{\mathrm{SiO}_{2}}^{\mathrm{liq}}\right)\right]-1.41 \mathrm{NM}+0.222 \mathrm{H}_{2} \mathrm{O}^{\text {liq }} \\
& +0.5 \mathrm{P}(\mathrm{GPa})\}
\end{aligned}
$$

donde:

$$
\begin{aligned}
& \mathrm{D}_{\mathrm{Mg}}^{\mathrm{O} / \mathrm{liq}}=\mathrm{X}_{\mathrm{Mg}}^{\mathrm{Ol}} / \mathrm{X}_{\mathrm{Mg}}^{\mathrm{liq}} \\
& \mathrm{C}_{\mathrm{NM}}^{\mathrm{L}}=\mathrm{X}_{\mathrm{FeO}}^{\mathrm{liq}}+X_{\mathrm{MnO}}^{\mathrm{liq}}+X_{\mathrm{MgO}}^{\mathrm{liq}}+X_{\mathrm{CaO}}^{\mathrm{liq}}+X_{\mathrm{CoO}}^{\mathrm{liq}}+X_{\mathrm{NiO}}^{\mathrm{liq}} \\
& \mathrm{C}_{\mathrm{SiO}_{2}}^{\mathrm{liq}}=X_{\mathrm{SiO}_{2}}^{\mathrm{liq}} \\
& \mathrm{NF}=\frac{7}{2} \ln \left(1-\mathrm{X}_{\mathrm{AlO}_{1.5}}^{\mathrm{liq}}\right)+7 \ln \left(1-\mathrm{X}_{\mathrm{TiO}_{2}}^{\mathrm{liq}}\right)
\end{aligned}
$$

Esta ecuación es esencialmente una modificación del modelo de Beattie (1993), basado en el reparto de $\mathrm{Mg}$ entre olivino y líquido, pero que evita las sobreestimaciones en el cálculo de $\mathrm{T}$ en condiciones hidratadas.

Para testar la existencia de equilibrio entre olivino y el líquido, se utiliza el coeficiente de distribución Fe-Mg de la reacción:

$$
\mathrm{MgO}^{\mathrm{Ol}}+\mathrm{FeO}^{\mathrm{liq}}=\mathrm{MgO}^{\mathrm{liq}}+\mathrm{FeO}^{\mathrm{Ol}}
$$

cuya expresión es:

$$
\mathrm{K}_{\mathrm{D}}(\mathrm{Fe}-\mathrm{Mg})^{\mathrm{Ol}-\mathrm{liq}}=\left(\mathrm{X}_{\mathrm{Fe}}^{\mathrm{Ol}} * \mathrm{X}_{\mathrm{Mg}}^{\mathrm{liq}}\right) /\left(\mathrm{X}_{\mathrm{Mg}}^{\mathrm{Ol}} * \mathrm{X}_{\mathrm{Fe}}^{\mathrm{liq}}\right)
$$

Como demostraron Roeder \& Emslie (1970), este parámetro varía muy poco con la $\mathrm{T}$ o la composición del par olivino-líquido y tiene un valor prácticamente constante de $0.30 \pm 0.03$ en sistemas basálticos a $\mathrm{P}<2-3 \mathrm{GPa}$.

\section{Plagioclasa}

En el caso de la plagioclasa, teniendo en cuenta la habitual presencia de agua en rocas calcoalcalinas (típicamente entre $1 \%$ y $4 \%$ en volcanes del CVMG; Johnson et al. 2010), hemos adoptado la ecuación 24a de Putirka (2008), que considera la posible presencia de agua en el líquido y reduce los errores respecto a calibraciones anteriores (p.ej. Ghiorso et al., 2002; Mathez, 1973; Putirka, 2005).

$$
\begin{aligned}
\frac{10^{4}}{\mathrm{~T}(\mathrm{~K})}= & 6.4706+0.3128 \ln \left\{\frac{\mathrm{X}_{\mathrm{An}}^{\mathrm{Pl}}}{\mathrm{X}_{\mathrm{CaO}}^{\mathrm{liq}}\left(\mathrm{X}_{\mathrm{AlO}_{1.5}}^{\mathrm{liq}}\right)^{2}\left(\mathrm{X}_{\mathrm{SiO}_{2}}^{\mathrm{liq}}\right)^{2}}\right\} \\
& -8.103\left(\mathrm{X}_{\mathrm{SiO}_{2}}^{\mathrm{liq}}\right)+4.87\left(\mathrm{X}_{\mathrm{KO}_{0.5} \mathrm{liq}}^{\mathrm{li}}\right)+1.5346\left(\mathrm{X}_{\mathrm{Ab}}^{\mathrm{Pl}}\right)^{2} \\
& +8.661\left(\mathrm{X}_{\mathrm{SiO}_{2}}^{\mathrm{liq}}\right)-3.341 * 10^{-2}(\mathrm{P}(\mathrm{kbar})) \\
& +0.18047\left(\mathrm{H}_{2} \mathrm{O}^{\mathrm{liq}}\right)
\end{aligned}
$$

Para los cálculos de presión hemos utilizado la ecuación. 25a de Putirka (2008), originaria de Putirka (2005), por coherencia con el termómetro.

$\mathrm{P}(\mathrm{kbar})=$

$$
\begin{aligned}
& -42.2+4.94 \times 10^{-2} \mathrm{~T}(\mathrm{~K})+1.16 \times 10^{-2} \mathrm{~T}(\mathrm{~K}) \\
& \ln \left\{\frac{\mathrm{X}_{\mathrm{Ab}}^{\mathrm{Pl}} \mathrm{X}_{\mathrm{AlO} \mathrm{O}_{1.5}}^{\mathrm{liq}} \mathrm{X}_{\mathrm{CaO}}^{\mathrm{liq}}}{\mathrm{X}_{\mathrm{An}}^{\mathrm{Pl}} \mathrm{X}_{\mathrm{NaO}_{0.5}}^{\mathrm{liq}} \mathrm{X}_{\mathrm{SiO}_{2}}^{\mathrm{liq}}}\right\}-382.3\left(\mathrm{X}_{\mathrm{SiO}_{2}}^{\mathrm{liq}}\right)^{2} \\
& +514.2\left(\mathrm{X}_{\mathrm{SiO}_{2}}^{\mathrm{liq}}\right)^{3}-19.6 \ln \left(\mathrm{X}_{\mathrm{Ab}}^{\mathrm{Pl}}\right)-139.8\left(\mathrm{X}_{\mathrm{CaO}}^{\mathrm{liq}}\right) \\
& +287.2\left(\mathrm{X}_{\mathrm{NaO}_{0.5}}^{\mathrm{liq}}\right)+163.9\left(\mathrm{X}_{\mathrm{KO}_{0.5}}^{\mathrm{liq}}\right)
\end{aligned}
$$

Como prueba de equilibrio del par Pl-liq se ha utilizado la constante de equilibrio $\mathrm{K}_{\mathrm{D}}(\mathrm{An}-\mathrm{Ab})^{\mathrm{P}-\text { liq }}$. Aunque este parámetro varía respecto a $\mathrm{P}, \mathrm{T}_{\mathrm{y}} \mathrm{H}_{2} \mathrm{O}$, al ser dividido en dos intervalos de $\mathrm{T}$ se obtienen 
valores relativamente constantes de $\mathrm{K}_{\mathrm{D}}(\mathrm{An}-\mathrm{Ab})$ Pl-liq $=0,10 \pm 0,05$ para $\mathrm{T}<1050{ }^{\circ} \mathrm{C}$ y $\mathrm{K}_{\mathrm{D}}(\mathrm{An}-\mathrm{Ab})$ ${ }^{\text {Pl-liq }}=0,27 \pm 0,11$ para $\mathrm{T}>1050^{\circ} \mathrm{C}$.

También se ha empleado la expresión $25 \mathrm{~b}$ de (Putirka, 2008) ya que el equilibrio Pl-líquido proporciona un buen higrómetro cuando $\mathrm{T}$ es conocida:

$$
\begin{aligned}
& \mathrm{H}_{2} \mathrm{O}(\mathrm{Wt})=25.95-0.0032 \mathrm{~T}\left({ }^{\circ} \mathrm{C}\right) \\
& \ln \left\{\frac{\mathrm{X}_{\mathrm{An}}^{\mathrm{Pl}}}{\mathrm{X}_{\mathrm{CaO}}^{\mathrm{liq}}\left(\mathrm{X}_{\mathrm{AlO}_{1.5}}^{\mathrm{liq}}\right)^{2}\left(\mathrm{X}_{\mathrm{SiO}_{2}}^{\mathrm{liq}}\right)^{2}}\right\}-18.9\left(\mathrm{X}_{\mathrm{KO}_{0.5}}^{\mathrm{liq}}\right)+14.5\left(\mathrm{X}_{\mathrm{MgO}}^{\mathrm{liq}}\right) \\
& -40.3\left(\mathrm{X}_{\mathrm{CaO}}^{\mathrm{liq}}\right)+5.7\left(\mathrm{X}_{\mathrm{An}}^{\mathrm{pl}}\right)+0.108 \mathrm{P}(\mathrm{Kbar})
\end{aligned}
$$

Puesto que estas expresiones requieren conocer previamente algunos parámetros de las otras, la resolución se ha efectuado mediante cálculo iterativo.

\section{Ortopiroxeno}

Para estimar las temperaturas de cristalización del ortopiroxeno hemos adoptado el termómetro calibrado por Putirka (2008) en su ecuación 28a. Esta expresión rectifica algunos problemas de sobreestimación de $\mathrm{T}$ asociados con el anterior termómetro de Beattie (1993), que hasta entonces era el único disponible, basado en el equilibrio Opx-líquido. La nueva calibración es aplicable en un amplio rango de $\mathrm{P}$ y entre $750-1600{ }^{\circ} \mathrm{C}, \mathrm{SiO}_{2}=33-77 \%$ y $\mathrm{H}_{2} \mathrm{O}=0-14.2 \%$, con un error $\mathrm{de} \pm 39^{\circ} \mathrm{C}(\mathrm{n}=793)$.

$$
\begin{aligned}
& \frac{10^{4}}{\mathrm{~T}\left({ }^{\circ} \mathrm{C}\right)}=4.07-0.329[\mathrm{P}(\mathrm{GPa})]+0.12\left[\mathrm{H}_{2} \mathrm{O}^{\mathrm{liq}}\right] \\
& +0.567 \ln \left[\frac{\mathrm{X}_{\mathrm{Fm}_{2} \mathrm{Sii}_{2} \mathrm{O}_{6}}^{\mathrm{Opx}^{2}}}{\left(\mathrm{X}_{\mathrm{SiO}_{2}}^{\mathrm{liq}}\right)^{2}\left(\mathrm{X}_{\mathrm{FeO}}^{\mathrm{liq}}+\mathrm{X}_{\mathrm{MnO}}^{\mathrm{liq}}+\mathrm{X}_{\mathrm{MgO}}^{\mathrm{liq}}\right)^{2}}\right] \\
& -3.06\left[\mathrm{X}_{\mathrm{MgO}}^{\mathrm{liq}}\right]-6.17\left[\mathrm{X}_{\mathrm{KO}_{0.5}}^{\mathrm{liq}}\right]+\left(\mathrm{Mg} \#^{\mathrm{liq}}\right)+2.57\left[\mathrm{X}_{\mathrm{Fe}}^{\mathrm{Opx}}\right]
\end{aligned}
$$

donde $\mathrm{Fm}=\mathrm{Fe}+\mathrm{Mn}+\mathrm{Mg}$

Para los cálculos de $\mathrm{P}$ hemos seleccionado la ecuación 29a de Putirka (2008), que utiliza la correlación positiva de $\mathrm{FmAl}_{2} \mathrm{SiO}_{6}$ respecto a $\mathrm{P}$ (entre $0.001 \mathrm{y}$ $0.05 \mathrm{kbar}$ ) y proporciona estimaciones con un error de \pm 2.6 kbar.
$\mathrm{P}(\mathrm{kbar})=$

$-13.97+0.0129 \mathrm{~T}\left({ }^{\circ} \mathrm{C}\right)+0.001416 \mathrm{~T}\left({ }^{\circ} \mathrm{C}\right)$

$\ln \left[\frac{\mathrm{X}_{\mathrm{NaAlSiO}_{2} \mathrm{O}_{6}}^{\mathrm{Opx}}}{\mathrm{X}_{\mathrm{NaO}_{0.5}}^{\mathrm{liq}} \mathrm{X}_{\mathrm{AlO}_{1.5}}^{\mathrm{liq}}\left(\mathrm{X}_{\left.\mathrm{SiO}_{2}\right)^{\mathrm{liq}}}^{2}\right.}\right]-19.64\left(\mathrm{X}_{\mathrm{SiO}_{2}}^{\mathrm{liq}}\right)$

$+47.49\left(\mathrm{X}_{\mathrm{MgO}}^{\mathrm{liq}}\right)+6.99\left(\mathrm{X}_{\mathrm{Fe}}^{\mathrm{Opx}}\right)+37.37\left(\mathrm{X}_{\mathrm{FmAl}_{2} \mathrm{SiO}_{6}}^{\mathrm{Opx}}\right)$

$+0.748\left(\mathrm{H}_{2} \mathrm{O}^{\mathrm{liq}}\right)+79.67\left(\mathrm{X}_{\mathrm{NaO}_{0.5}}^{\mathrm{liq}}+\mathrm{X}_{\mathrm{KO}_{0.5}}^{\mathrm{liq}}\right)$

Como test de equilibrio del par Opx-líquido nos basamos en el diagrama de Rhodes (Rhodes et al., 1979) y el valor $K_{D}(F e-M g)^{\text {Opx-liq }}=0.29 \pm 0.06$ (para $\mathrm{n}=785$ ). Este valor no depende de P y T, pero disminuye ligeramente con el aumento en contenido en sílice de modo que $\mathrm{K}_{\mathrm{D}}(\mathrm{Fe}-\mathrm{Mg})^{\mathrm{Opx}-\mathrm{liq}}=$ $0,4805-0,3733 * \mathrm{X}_{\mathrm{Si}}^{\text {liq }}$.

\section{Clinopiroxeno}

Los barómetros para clinopiroxenos se basan en la reacción $\mathrm{NaO}_{0.5}^{\text {liq }}+\mathrm{AlO}_{1.5}^{\text {liq }}+2 \mathrm{SiO}_{2}^{\text {liq }}=$ $\mathrm{NaAlSi}_{2} \mathrm{O}_{6}$ cpx , si bien los modelos más recientes han sido calibrados usando $\mathrm{H}_{2} \mathrm{O}^{\text {liq }}$ (en \% en peso) como variable para describir mejor las muestras hidratadas (Grove \& Juster, 1989; Kinzler \& Grove, 1992; Patiño Douce, 2005; Putirka et al., 1996; Putirka, 2008; Scaillet \& MacDonald, 2003; Sisson \& Grove, 1993a; Sisson \& Grove, 1993b; Walter \& Presnall, 1994). De estas, hemos seleccionado para el cálculo de $\mathrm{P}$ la expresión $32 \mathrm{c}$ de Putirka (2008), por su buen ajuste respecto a valores experimentales (error estimado de $\pm 1.5 \mathrm{kbar}$ para $n=99$ ).

$$
\begin{aligned}
\mathrm{P}(\text { kbar })= & -57.9+0.0475 \mathrm{~T}(\mathrm{~K})-40.6\left(\mathrm{X}_{\mathrm{FeO}}^{\mathrm{liq}}\right)-47.7\left(\mathrm{X}_{\mathrm{CaTs}}^{\mathrm{Cpx}}\right) \\
& +0.676\left(\mathrm{H}_{2} \mathrm{O}^{\mathrm{liq}}\right)-153\left(\mathrm{X}_{\mathrm{CaO}_{0.5}}^{\mathrm{liq}} \mathrm{X}_{\mathrm{SiO}_{2}}^{\mathrm{liq}}\right)+6.89\left[\frac{\mathrm{X}_{\mathrm{Al}}^{\mathrm{Cpx}}}{\mathrm{X}_{\mathrm{AlO}_{1.5}}^{\mathrm{lq}}}\right]
\end{aligned}
$$

Análogamente, para los cálculos de temperatura se ha utilizado la ecuación 33 de (Putirka, 2008), con errores estimados de $\pm 42^{\circ} \mathrm{C}$ (para $\mathrm{n}=320$ hidratado) $\mathrm{y} \pm 46^{\circ} \mathrm{C}$ (para $\mathrm{n}=854$ anhidro). 


$$
\begin{aligned}
\frac{10^{4}}{\mathrm{~T}(\mathrm{~K})}= & 7.53-0.14 \ln \left(\frac{\mathrm{X}_{\mathrm{ld}}^{\mathrm{Cpx}} \mathrm{X}_{\mathrm{CaO}}^{\mathrm{liq}} \mathrm{X}_{\mathrm{Fm}}^{\mathrm{liq}}}{\mathrm{X}_{\mathrm{DiHd}}^{\mathrm{Cpx}} X_{\mathrm{Na}}^{\mathrm{liq}} \mathrm{X}_{\mathrm{Al}}^{\mathrm{liq}}}\right)+0.07\left(\mathrm{H}_{2} \mathrm{O}^{\mathrm{liq}}\right) \\
& -14.9\left(\mathrm{X}_{\mathrm{CaO}}^{\mathrm{liq}} \mathrm{X}_{\mathrm{SiO}_{2}}^{\mathrm{liq}}\right)-0.08 \ln \left(\mathrm{X}_{\mathrm{TiO}_{2}}^{\mathrm{liq}}\right) \\
& -3.62\left(\mathrm{X}_{\mathrm{NaO}_{0.5}}^{\mathrm{liq}}+\mathrm{X}_{\mathrm{KO}}^{\mathrm{liq}}\right)-1.1\left(\mathrm{Mg} \#^{\mathrm{liq}}\right) \\
& -0.18 \ln \left(\mathrm{X}_{\mathrm{EnFs}}^{\mathrm{Cpx}}\right)-0.027 \mathrm{P}(\mathrm{kbar})
\end{aligned}
$$

Como test de equilibrio se ha calculado el valor de $\mathrm{K}_{\mathrm{D}}(\mathrm{Fe}-\mathrm{Mg})^{\mathrm{Cpx}-\mathrm{liq}}$, que adopta un valor de $0,28 \pm 0,08$ mostrando poca variación respecto a la temperatura (Putirka, 2008).

\section{Anfíbol}

Para calcular los valores de P y T de cristalización de los anfíboles hemos adoptado el reciente termobarómetro de Ridolfi et al. (2010), que no se basa en el equilibrio con el líquido y representa el único modelo actualmente disponible para anfíboles en magmas calcoalcalinos. Esta expresión permite asimismo calcular el porcentaje de $\mathrm{H}_{2} \mathrm{O}$ del magma a partir del cual cristalizaría el anfíbol analizado.

$T=-151.487 S i^{*}+2,041$

donde:

$$
\begin{aligned}
\mathrm{Si}^{*}= & \mathrm{Si}+\frac{{ }^{[4]} \mathrm{Al}}{15}-2^{[4]} \mathrm{Ti}-\frac{{ }^{[6]} \mathrm{Al}}{2}-\frac{{ }^{[6]} \mathrm{Al}}{1.8}+\frac{\mathrm{Fe}^{3+}}{9}+\frac{\mathrm{Fe}^{2+}}{3.3} \\
& +\frac{\mathrm{Mg}}{26}+\frac{{ }^{\mathrm{B}} \mathrm{Ca}}{5}+\frac{{ }^{\mathrm{B}} \mathrm{Na}}{1.3}-\frac{{ }^{\mathrm{A}} \mathrm{Na}}{15}+\frac{{ }^{\mathrm{A}}[]}{2.3}
\end{aligned}
$$

$\left.\mathrm{T}=19.209 \mathrm{e}^{(1.438 \mathrm{Al}} \mathrm{I}_{\mathrm{T}}\right)$

donde $\mathrm{Al}_{\mathrm{T}}$ es aluminio total.

$\mathrm{H}_{2} \mathrm{O}_{\text {melt }}=5.215^{[6]} \mathrm{Al}^{*}+12.28$

donde:

$$
\begin{aligned}
& { }^{[6]} \mathrm{Al}^{*}={ }^{[6]} \mathrm{Al}+\frac{{ }^{[6]} \mathrm{Al}}{13.9}-\frac{\mathrm{Si}+{ }^{[6]} \mathrm{Ti}}{5}-\frac{{ }^{\mathrm{c}} \mathrm{Fe}^{2+}}{3} \\
& -\frac{\mathrm{mg}}{1.7}+\frac{\mathrm{Ca}+{ }^{\mathrm{A}}[]}{1.2}+\frac{{ }^{\mathrm{A}} \mathrm{Na}}{2.7}-1.56 \mathrm{~K}-\frac{\mathrm{Fe} \#}{1.6}
\end{aligned}
$$

\section{Resultados}

Como se ha indicado anteriormente, la aplicación de geotermómetros basados en equilibrios minerallíquido plantea ciertas limitaciones ya que requiere contar con pares que no muestren signos de desequilibrio (p.ej. bordes corroídos o coronas de reacción). Además, si se acepta que la composición del líquido está representada por la roca total, es necesario asumir que el equilibrio se verifica únicamente con el borde de los minerales presentes en la roca. En consecuencia, las temperaturas obtenidas se limitan a la etapa final de cristalización de dichas fases. Para obtener las temperaturas a las que se produjo la cristalización de zonas internas de los minerales, sería necesario conocer la composición del líquido en equilibrio en el momento de la cristalización de dichas zonas.

De acuerdo con lo expuesto, en este trabajo los cálculos basados en geotermómetros mineral-líquido se han restringido a aquellos análisis obtenidos en minerales que no muestran evidencias texturales de desequilibrio respecto a la matriz y limitados bien a minerales que no muestran zonación (usualmente microcristales), o bien a bordes de fenocristales o microfenocristales. Asimismo, como se ha indicado más arriba, se han considerado únicamente aquellos pares mineral-líquido que proporcionan valores $\mathrm{K}_{\mathrm{D}}$ dentro de los rangos considerados de equilibrio en cada caso. En las Tablas 2 a 6 y en la fig. 6 se recogen los resultados obtenidos de esta aproximación.

En el caso del Ol (Tabla 2), las temperaturas obtenidas oscilan entre $1232-1198^{\circ} \mathrm{C}$, con una tendencia general a disminuir la temperatura de cristalización a medida que progresa la diferenciación (Fig.6A).

Los cálculos para equilibrios Pl-liq proporcionan unas temperaturas y presiones de $1162-1126{ }^{\circ} \mathrm{C}$, y 7.3-3.6 kbar respectivamente (Tabla 3). No se han observado diferencias significativas en los valores de $\mathrm{T}$ respecto al contenido en $\mathrm{SiO}_{2}$ entre los distintos tipos de plagioclasas ni entre fenocristales y microcristales. Por otro lado, la variación de P respecto a $\mathrm{T}$ sugiere un rango de presiones bastante más amplio que el que se observa en las temperaturas calculadas.

Por su parte, la cristalización de los ortopiroxenos abarca un rango de temperaturas entre $1147^{\circ} \mathrm{C}$ y $1027^{\circ} \mathrm{C}$ (Tabla 4), con una tendencia general a la 


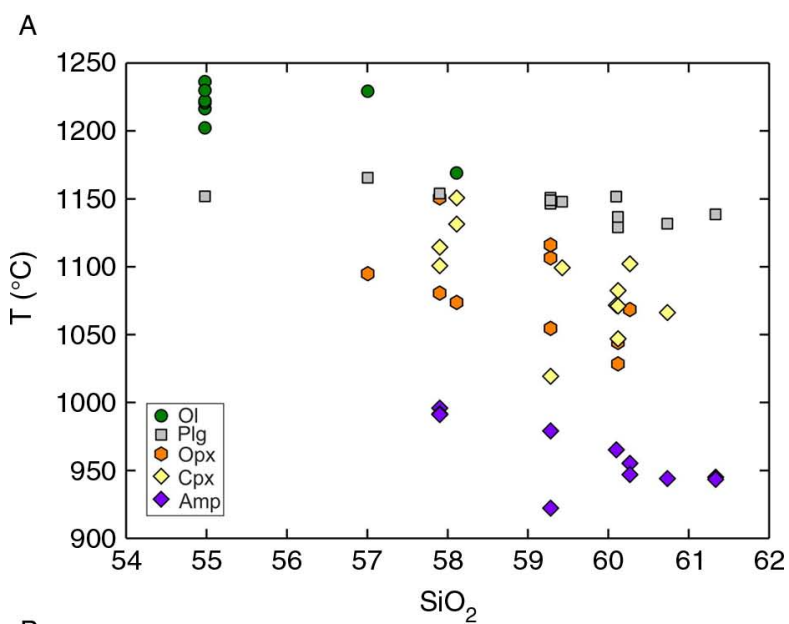

B

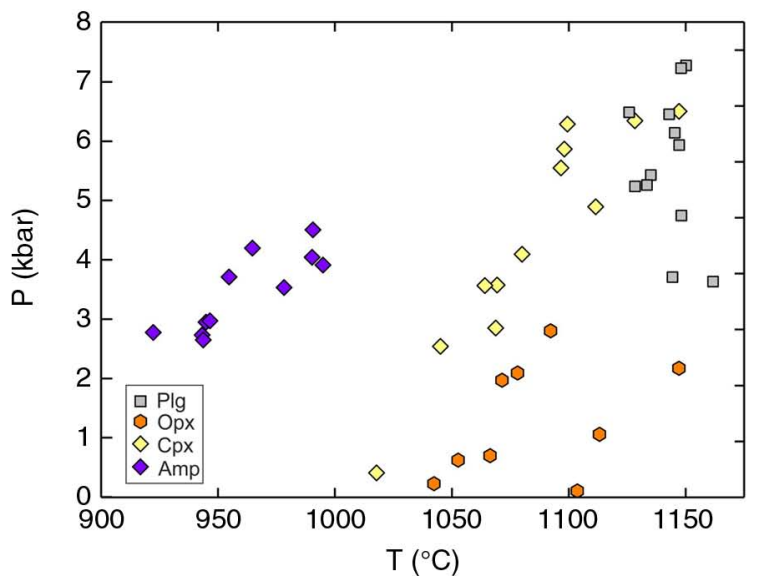

Fig. 6.-A) Diagrama de temperatura vs. $\mathrm{SiO} 2$ (\% en peso) en roca total; y B) Diagrama de presión vs temperatura obtenidos para olivino, plagioclasa, ortopiroxeno, clinopiroxeno y anfíbol en las lavas de El Metate.

$\mathrm{T}$ al aumentar el contenido de $\mathrm{SiO}_{2}$ (Fig. 6A). En el gráfico $\mathrm{P}-\mathrm{T}$ los resultados se disponen con pocas excepciones en una relación directa de disminución de $\mathrm{P}$ con respecto a $\mathrm{T}$ (Fig. 6B). Como en el caso de las plagioclasas, no se observan diferencias entre las dos trayectorias de evolución o los tipos de Opx identificados.

El clinopiroxeno presenta el rango más amplio de temperaturas, desde los $1147^{\circ} \mathrm{C}$ a los $1018^{\circ} \mathrm{C}$ (Tabla 5), con cierta tendencia a disminuir el valor de $\mathrm{T}$ con el aumento de $\mathrm{SiO}_{2}$ (Fig. 6A). La variación de $\mathrm{P}$ con respecto a la $\mathrm{T}$ sigue, como en el resto de fases analizadas, la relación directa habitual. Al igual que en el caso de Pl y Opx, tampoco se observan diferencias significativas en función de las diferentes tipologías de cristales analizados o de las pautas de evolución identificadas a partir de elementos mayores en roca total.

Los anfíboles analizados indican un rango de variación de $\mathrm{T}$ para la cristalización de esta fase entre 995 y $922{ }^{\circ} \mathrm{C}$ (Tabla 6), disponiéndose en una pauta de pendiente negativa respecto al aumento del contenido en $\mathrm{SiO}_{2}$ de la roca correspondiente (Fig. 6A). Tanto los núcleos como los bordes analizados se proyectan en todo el rango de variación de $\mathrm{T}-\mathrm{SiO}_{2}$, aunque en este caso se aprecia cierta disminución de $\mathrm{T}$ de centro a borde en los cristales zonados. Por otro lado, las muestras con menor $\mathrm{SiO}_{2}$ presentan los valores de $\mathrm{P}$ más altos, siguiendo una tendencia $\mathrm{P}-\mathrm{T}$ análoga al resto de fases minerales (Fig. 6B).

\section{Discusión y conclusiones}

Como se desprende de las variaciones composicionales basadas en elementos mayores, puestas de manifiesto en los diagramas $\mathrm{SiO}_{2}$-elemento (Fig. 4), las lavas de El Metate se han fraccionado a partir de dos pautas de diferenciación constrastadas. Sin embargo, por lo que respecta a sus características petrográficas, apenas se observan diferencias significativas entre ambas tendencias. Así, la presencia de olivino, que está limitada a una de las pautas de evolución, coincide con los términos de menor grado de diferenciación (es decir, con mayor $\mathrm{MgO}$ y \#Mg, y menor $\mathrm{SiO}_{2}$ ), que están ausentes en la otra línea de diferenciación. Asimismo, si bien la presencia de anfíbol es característica de la serie con menor abundancia de $\mathrm{MgO}$, dicha fase también se encuentra en las rocas con mayor grado de diferenciación del otro grupo (y por ende, con menor contenido relativo en $\mathrm{MgO}$ y $\mathrm{K}_{2} \mathrm{O}$ ). A pesar de estas diferencias, en todos los casos P1, Opx y Cpx, aparecen como fenocristales y por tanto deben haber participado en el proceso de diferenciación de ambas series, sin que tampoco se hayan detectado diferencias significativas en lo que se refiere a la composición o la textura de ninguna de las fases estudiadas entre ambos grupos de rocas.

Por lo que respecta a las características del proceso de diferenciación, la frecuente presencia de texturas de desequilibrio en muchas de las fases (p.ej. zonaciones complejas, bordes corroídos y recrecimientos en plagioclasas, bordes de opacita y coronas de reacción en anfíboles), sugieren una evolución relativamente compleja. La posibilidad 
más razonable, siendo además el escenario más habitualmente observado en el volcanismo monogenético de Michoacán (ver p.ej. Cebriá et al., 2011; Corona-Chávez et al., 2006; Chesley et al., 2002; Luhr \& Carmichael, 1985; Urrutia-Fucugauchi \& Uribe-Cifuentes, 1999), sugiere a una evolución en sistema abierto, que podría implicar tanto la asimilación de material xenolítico como inyecciones de nuevos aportes de magma en el sistema. Por este motivo resulta especialmente adecuada la aproximación adoptada aquí, considerando exclusivamente aquellos minerales que representan fases en equilibrio con el magma que los engloba y cuya composición se asume respresentada por la composición de la roca total. Obviamente, esto implica que los resultados termobarométricos obtenidos se refieren al momento final de la cristalización de cada fase y por tanto es posible que no aporten información sobre las etapas previas de cristalización, cuyo estudio requeriría establecer la composición de los respectivos líquidos en equilibrio.

En la fig. 6A se presentan las temperaturas obtenidas para cada una de las fases respecto al contenido en $\mathrm{SiO}_{2}$ de la roca, que se puede asumir como indicador del grado de diferenciación. Como se puede observar, la secuencia de cristalización se verifica en un relativamente estrecho margen de temperatura, comenzando con la aparición de $\mathrm{Ol}$ $\left(1232-1198{ }^{\circ} \mathrm{C}\right)$, seguido de Pl $\left(1162-1126{ }^{\circ} \mathrm{C}\right)$, Opx $\left(1147-1027{ }^{\circ} \mathrm{C}\right)$ y Cpx $\left(1147-1018{ }^{\circ} \mathrm{C}\right)$. En esta secuencia, el Ol deja de cristalizar con la aparición del Opx y los fenocristales de Pl con la del Cpx. Por su parte, el Amp cristalizaría en una última etapa, entre los $995^{\circ} \mathrm{C}$ y $922^{\circ} \mathrm{C}$.

Los datos de P obtenidos (Fig. 6B) por los diferentes geobarómetros, indican que la cristalización se verifica entre los $\sim 7$ y $0 \mathrm{kbar}$, con una correspondencia directa entre $\mathrm{P}$ y $\mathrm{T}$ lo que sugiere que el proceso de diferenciación tuvo lugar desde $\sim 25 \mathrm{~km}$ de profundidad hasta niveles superficiales.

Es de destacar que, como se ha descrito, los cristales de anfíbol presentan en todos los casos evidencias de desequilibrio, a pesar de lo cual se han podido calcular valores de T, $\mathrm{P}$ y contenido en agua gracias a la aplicación de un geotermobarómetro basado únicamente en la composición del mineral, por lo que estos datos solo aportan las condiciones de cristalización de los Amp, independientemente de que se encuentren o no en equilibrio con la roca huesped. En consecuencia, cabe la posibilidad de que los cristales de anfíbol representen xenocristales y que por tanto no se encuentren en equilibrio con el magma que los engloba. La procedencia de estos xenocristales podría resultar bien de la asimilación de las rocas atravesadas por el magma durante su ascenso a la superficie o bien de la mezcla con un pulso de magma más silíceo y de menor temperatura. Por otro lado, las condiciones geotermobarométricas calculadas y las variaciones en elementos mayores, también podrían explicarse si el Amp se genera en la última etapa en la secuencia de cristalización de estos magmas. Este supuesto se vería apoyado por la existencia de Amp en las dos tendencias de diferenciación identificadas, apareciendo siempre en los términos más evolucionados (con menor contenido en $\mathrm{MgO}$ ) y en relación directa con un característico empobrecimiento en $\mathrm{K}_{2} \mathrm{O}$ (que sería consecuencia del fraccionamiento de esta fase). Asimismo, esta hipotesis se vería reforzada ya que la cristalización de Amp está limitada a presiones entre 5 y 2.5 kbar. Esto podría explicar su desequilibrio al alcanzar niveles superficiales, donde se produce la desgasificación del magma y una reducción drástica del contenido en $\mathrm{H}_{2} \mathrm{O}$.

$\mathrm{Al}$ igual que en el caso del Amp, las evidencias de desequilibrio de otras fases pueden explicarse por desestabilización de dichas fases tras su cristalización durante etapas previas de la diferenciación, al variar las condiciones fisicoquímicas del magma en etapas posteriores de su evolución. Por ejemplo, en el caso de la Pl la desestabilización puede ser debida al incremento en el contenido de agua durante la cristalización, que como hemos visto se sitúa en torno al $1.6 \%$ durante su cristalización y alcanzaría hasta el 6.9\% durante la cristalización de Amp. Esta es una situación relativamente frecuente en magmas calcoalcalinos y se ha observado también en volcanes cercanos como el Paricutin (Cebriá et al., 2011), donde su desaparición como fenocristal coincidiendo con la aparición de Opx, se interpretó como consecuencia del aumento en $\mathrm{H}_{2} \mathrm{O}$, en este caso debido a la entrada de agua en el sistema por asimilación de un componente cortical.

En resumen, los datos preliminares obtenidos en este estudio permiten establecer las condiciones 
P-T- $\mathrm{H}_{2} \mathrm{O}$ bajo las que ha tenido lugar el proceso de diferenciación de las lavas de El Metate. Así, la secuencia de cristalización $\mathrm{Ol} \pm \mathrm{Pl}-\mathrm{Opx}$ Cpx(土Amp?) parece iniciarse a unos $25 \mathrm{~km}$ de profundidad y temperaturas en torno a $1230{ }^{\circ} \mathrm{C}$. El aumento del contenido en $\mathrm{H}_{2} \mathrm{O}$ que se produce en el sistema al progresar la cristalización pudo haber provocado en primer lugar que la Pl deje de ser una fase estable en el sistema y que cuando en torno a los $990^{\circ} \mathrm{C}$ se alcanza un porcentaje de agua de $\sim 7 \%$ se inicie la cristalización del Amp, que dejará de ser estable a niveles superficiales. No obstante las texturas de desequilibrio que presentan estas dos fases minerales sugieren que algunos cristales de $\mathrm{Pl}$ y los de Amp pueden tener un origen xenolítico.

Estos datos preliminares constituyen la base necesaria para perfilar un modelo petrogenético, que permita definir con precisión los parámetros del proceso, pero requieren resolver previamente algunas de las incóginas planteadas y que se encuentran actualmente en estudio. Así por ejemplo, es necesario establecer precisiones sobre las variaciones composicionales y termobarométricas a nivel de cristal en aquellos casos donde se observan zonaciones, lo que requiere además establecer la composición de sus correspondientes líquidos en equilibrio. Esto permitiría confirmar o descartar si en algún caso se trata de xenocristales y si se ha producido o no la entrada de nuevos aportes de magma en el sistema. Esta situación también podrá verificarse a partir del estudio de los microcristales presentes en coronas de reacción, como las observadas en Amp. Finalmente, contar con un mayor número de datos permitirá confirmar que realmente no existen diferencias termobarométricas entre las dos pautas de diferenciación observadas a partir de los elementos mayores de las rocas, como parecen indicar los primeros datos aquí expuestos.

\section{AGRADECIMIENTOS}

Este trabajo ha sido financiado mediante el proyecto CGL2011-23422 del Ministerio de Economía y Competitividad de España. Asimismo se agradece la concesión a E. Losantos de una beca FPI (BES-2012-052390) asignada a dicho proyecto.

Agradecemos a Rufino Lozano-Santa Cruz y Laura Luna González, del Instituto de Geología (UNAM) por los análisis de FRX y por el apoyo en los trabajos de campo respectivamente, y a Alfredo Fernández Larios del Centro Nacional de Microscopía
Electrónica (UCM) por su asistencia en los análisis por microsonda electrónica. Gracias especiales a Francisco Pérez Torrado (Universidad de Las Palmas de Gran Canaria) y a José Luis Arce (Instituto de Geología, UNAM) por la revisión del manuscrito original, que han mejorado el trabajo y han aportado nuevas ideas para futuras investigaciones.

\section{Referencias}

Beattie, P. (1993). Olivine-melt and orthopyroxene-melt equilibria. Contributions to Mineralogy and Petrology, 115(1): 103-111. http://dx.doi.org/10.1007/ BF00712982.

Cebriá, J.M.; Martiny, B.M.; López-Ruiz, J. \& MoránZenteno, D.J. (2011). The Parícutin calc-alkaline lavas: New geochemical and petrogenetic modelling constraints on the crustal assimilation process. Journal of Volcanology and Geothermal Research, 201(1-4): 113-125. http://dx.doi.org/10.1016/j.jvolgeores.2010. 11.011 .

Corona-Chávez, P.; Reyes-Salas, M.; Gardu-o-Monroy, V.H.; Israde-Alcántara, I.; Lozano-Santa Cruz, R.; Morton-Bermea, O. \& Hernández-Álvarez, E. (2006). Assimilation of granitic xenoliths in the MichoacánGuanajuato volcanic field: The case of Arócutin, Michoacán, Mexico. Revista Mexicana de Ciencias Geológicas, 23(2): 233-245.

Costa, F.; Andreastuti, S.; Bouvet de Maisonneuve, C. \& Pallister, J.S. (2013). Petrological insights into the storage conditions, and magmatic processes that yielded the centennial 2010 Merapi explosive eruption. Journal of Volcanology and Geothermal Research, 261: 209-235. http://dx.doi.org/10.1016/ j.jvolgeores.2012.12.025.

Chesley, J.; Ruiz, J.; Righter, K.; Ferrari, L. \& GómezTuena, A. (2002). Source contamination versus assimilation: An example from the Trans-Mexican volcanic arc. Earth and Planetary Science Letters, 195(3-4): 211-221. http://dx.doi.org/10.1016/S0012$821 \mathrm{X}(01) 00580-5$.

Dahren, B.; Troll, V.; Andersson, U.; Chadwick, J.; Gardner, M.; Jaxybulatov, K. \& Koulakov, I. (2012). Magma plumbing beneath Anak Krakatau volcano, Indonesia: evidence for multiple magma storage regions. Contributions to Mineralogy and Petrology, 163(4): 631-651. http://dx.doi.org/10.1007/s00410011-0690-8.

Ferrari, L.; López-Martínez, M.; Aguirre-Díaz, G. \& Carrasco-Núñez, G. (1999). Space-time patterns of Cenozoic arc volcanism in central Mexico: From the Sierra Madre Occidental to the Mexican Volcanic Belt. Geology, 27(4): 303-306. http:// dx.doi.org/10.1130/0091-7613(1999)027<0303: STPOCA $>2.3 . C O ; 2$.

Ferrari, L.; Orozco-Esquivel, T.; Manea, V. \& Manea, M. (2012). The dynamic history of the Trans-Mexican 
Volcanic Belt and the Mexico subduction zone. Tectonophysics, 522-523(0): 122-149. http://dx.doi.org/ 10.1016/j.tecto.2011.09.018.

Ghiorso, M.S.; Hirschmann, M.M.; Reiners, P.W. \& Kress, V.C. (2002). The pMELTS: A revision of MELTS for improved calculation of phase relations and major element partitioning related to partial melting of the mantle to $3 \mathrm{GPa}$. Geochemistry, Geophysics, Geosystems, 3(5): 1-35. http://dx.doi. org/10.1029/2001GC000217.

Gómez-Tuena, A.; Orozco-Esquivel, M.T. \& Ferrari, L. (2007). Igneous petrogenesis of the Trans-Mexican Volcanic Belt. In: Geology of México: Celebrating the Centenary of the Geological Society of México. (Alaniz-Álvarez, S.A.\& Nieto-Samaniego,A.F., eds.) Geological Society of America Special Paper 422, 129-181. http://dx.doi.org/10.1130/2007.2422(05).

Grove, T.L. \& Juster, T.C. (1989). Experimental investigations of low-Ca pyroxene stability and olivinepyroxene-liquid equilibria at 1-atm in natural basaltic and andesitic liquids. Contributions to Mineralogy and Petrology, 103(3): 287-305. http://dx.doi.org/10.1007/ BF00402916.

Hasenaka, T. (1994). Size, distribution, and magma output rate for shield volcanoes of the Michoacán-Guanajuato volcanic field, Central Mexico. Journal of Volcanology and Geothermal Research, 63(1-2): 13-31. http://dx.doi.org/10.1016/0377-0273(94)90016-7.

Hasenaka, T. \& Carmichael, I.S.E. (1985). The cinder cones of Michoacán-Guanajuato, central Mexico: their age, volume and distribution, and magma discharge rate. Journal of Volcanology and Geothermal Research, 25(1-2): 105-124. http://dx.doi.org/10. 1016/0377-0273(85)90007-1.

Hasenaka, T. \& Carmichael, I.S.E. (1987). The Cinder Cones of Michoacán-Guanajuato, Central Mexico: Petrology and Chemistry. Journal of Petrology, 28(2): 241-269. http://dx.doi.org/10.1093/petrology/28.2.241.

Johnson, C.A. \& Harrison, C.G.A. (1989). Tectonics and volcanism in Central Mexico: A landsat thematic mapper perspective. Remote Sensing of Environment, 28(0): 273-286. http://dx.doi.org/10.1016/ 0034-4257(89)90119-3.

Johnson, E.R.; Wallace, P.J.; Cashman, K.V. \& Delgado Granados, H. (2010). Degassing of volatiles $\left(\mathrm{H}_{2} \mathrm{O}\right.$, $\mathrm{CO}_{2}, \mathrm{~S}, \mathrm{Cl}$ ) during ascent, crystallization, and eruption at mafic monogenetic volcanoes in central Mexico. Journal of Volcanology and Geothermal Research, 197(1-4): 225-238. http://dx.doi.org/10. 1016/j.jvolgeores.2010.02.017.

Keiding, J.K. \& Sigmarsson, O. (2012). Geothermobarometry of the 2010 Eyjafjallajökull eruption: New constraints on Icelandic magma plumbing systems. Journal of Geophysical Research: Solid Earth, 117 (B9): B00C09.

Kinzler, R.J. \& Grove, T.L. (1992). Primary magmas of mid-ocean ridge basalts 1 . Experiments and methods.
Journal of Geophysical Research, 97(B5): 6885 6906. http://dx.doi.org/10.1029/91JB02840.

Le Bas, M.J.; Le Maitre, R.W. \& Woolley, A.R. (1992). The construction of the Total Alkali-Silica chemical classification of volcanic rocks. Mineralogy and Petrology, 46(1): 1-22. http://dx.doi.org/10.1007/ BF01160698.

Lozano-Santa Cruz, R. \& Bernal, J.P. (2005). Characterization of a new set of eight geochemical reference materials for XRF major and trace element analysis. Revista Mexicana de Ciencias Geológicas, 22(3): 329-344.

Luhr, J.F. \& Carmichael, I. (1985). Jorullo Volcano, Michoacán, Mexico (1759-1774): The earliest stages of fractionation in calc-alkaline magmas. Contributions to Mineralogy and Petrology, 90(2): 142-161. http://dx.doi.org/10.1007/BF00378256.

Mathez, E. (1973). Refinement of the Kudo-Weill plagioclase thermometer and its application to basaltic rocks. Contributions to Mineralogy and Petrology, 41(1): 61-72. http://dx.doi.org/10.1007/BF00377654.

Patiño Douce, A.E. (2005). Vapor-absent melting of tonalite at 15-32 kbar. Journal of Petrology, 46(2): 275-290. http://dx.doi.org/10.1093/petrology/egh071.

Plechov, P.Y.; Tsai, A.E.; Shcherbakov, V.D. \& Dirksen, O.V. (2008). Opacitization conditions of hornblende in Bezymyannyi volcano andesites (March 30, 1956 eruption). Petrology, 16(1): 19-35. http://dx.doi.org/ $10.1134 / \mathrm{S} 0869591108010025$.

Putirka, K.; Johnson, M.; Kinzler, R.; Longhi, J. \& Walker, D. (1996). Thermobarometry of mafic igneous rocks based on clinopyroxene-liquid equilibria, 0-30 kbar. Contributions to Mineralogy and Petrology, 123(1): 92-108. http://dx.doi.org/10.1007/s004100050145.

Putirka, K.D. (2005). Igneous thermometers and barometers based on plagioclase + liquid equilibria: Tests of some existing models and new calibrations. American Mineralogist, 90(2-3): 336-346. http://dx.doi. org/10.2138/am.2005.1449.

Putirka, K.D. (2008). Thermometers and Barometers for Volcanic Systems. Reviews in Mineralogy and Geochemistry, 69(1): 61-120. http://dx.doi.org/10.2138/ rmg.2008.69.3.

Rhodes, J.M.; Dungan, M.A.; Blanchard, D.P. \& Long, P.E. (1979). Magma mixing at mid-ocean ridges: Evidence from basalts drilled near $22^{\circ} \mathrm{N}$ on the midAtlantic Ridge. Tectonophysics, 55(1-2): 35-61. http://dx.doi.org/10.1016/0040-1951(79)90334-2.

Ridolfi, F.; Renzulli, A. \& Puerini, M. (2010). Stability and chemical equilibrium of amphibole in calc-alkaline magmas: an overview, new thermobarometric formulations and application to subduction-related volcanoes. Contributions to Mineralogy and Petrology, 160(1): 45-66. http://dx.doi.org/10.1007/s00410-0090465-7.

Roeder, P.L. \& Emslie, R.F. (1970). Olivine-liquid equilibrium. Contributions to Mineralogy and Petrology, 29: 275-289. http://dx.doi.org/10.1007/BF00371276. 
Scaillet, B. \& MacDonald, R. (2003). Experimental constraints on the relationships between peralkaline rhyolites of the Kenya Rift Valley. Journal of Petrology, 44(10): 1867-1894. http://dx.doi.org/10.1093/ petrology/egg062.

Sisson, T.W. \& Grove, T.L. (1993a). Experimental investigations of the role of $\mathrm{H}_{2} \mathrm{O}$ in calc-alkaline differentiation and subduction zone magmatism. Contributions to Mineralogy and Petrology, 113(2): 143-166. http://dx.doi.org/10.1007/BF00283225.

Sisson, T.W. \& Grove, T.L. (1993b). Temperatures and $\mathrm{H} 2 \mathrm{O}$ contents of low-MgO high-alumina basalts. Contributions to Mineralogy and Petrology, 113(2): 167-184. http://dx.doi.org/10.1007/BF00283226.

Streck, M.J. (2008). Mineral Textures and Zoning as Evidence for Open System Processes. Reviews in Mineralogy and Geochemistry, 69(1): 595-622. http:// dx.doi.org/10.2138/rmg.2008.69.15.
Suter, M.; Contreras, J.; Gómez-Tuena, A.; Siebe, C.; Quintero-Legorreta, O.; García-Palomo, A.; Macías, J.L.; Alaniz-Álvarez, S.A.; Nieto-Samaniego, A.F. \& Ferrari, L. (1999). Effect of strain rate in the distribution of monogenetic and polygenetic volcanism in the Transmexican volcanic belt: Comments and Reply. Geology, 27(6): 571-575. http://dx.doi.org/10.1130/ 0091-7613(1999)027<0571:EOSRIT>2.3.CO;2.

Urrutia-Fucugauchi, J. \& Uribe-Cifuentes, R.M. (1999). Lower-crustal xenoliths from the Valle de Santiago maar field, Michoacan-Guanajuato volcanic field, central Mexico. International Geology Review, 41(12): 10671081. http://dx.doi.org/10.1080/00206819909465192.

Walter, M.J. \& Presnall, D.C. (1994). Melting behavior of simplified lherzolite in the system CaO-MgO$\mathrm{Al}_{2} \mathrm{O}_{3}-\mathrm{SiO}_{2}-\mathrm{Na}_{2} \mathrm{O}$ from 7 to 35 kbar. Journal of Petrology, 35(2): 329-359. http://dx.doi.org/10.1093/ petrology/35.2.329. 